\title{
Classical Yang-Mills observables from amplitudes
}

\section{Leonardo de la Cruz, Ben Maybee, Donal O'Connell and Alasdair Ross}

Higgs Centre for Theoretical Physics, School of Physics and Astronomy, University of Edinburgh, Peter Guthrie Tate Road, Edinburgh, EH9 3FD, Scotland, U.K.

E-mail: leonardo.delacruz@ed.ac.uk, b.maybee@ed.ac.uk, donal@staffmail.ed.ac.uk, alasdair.ross@ed.ac.uk

ABSTRACT: The double copy suggests that the basis of the dynamics of general relativity is Yang-Mills theory. Motivated by the importance of the relativistic two-body problem, we study the classical dynamics of colour-charged particle scattering from the perspective of amplitudes, rather than equations of motion. We explain how to compute the change of colour, and the radiation of colour, during a classical collision. We apply our formalism at next-to-leading order for the colour change and at leading order for colour radiation.

KEYwords: Scattering Amplitudes, Gauge Symmetry

ArXIV EPRINT: 2009.03842 


\section{Contents}

1 Introduction 1

2 Review of the theory of colour 3

2.1 Colour in classical field theory 4

2.2 Colour of single-particle quantum states 5

2.3 Colour of point-like particles in the classical regime $\quad 7$

$\begin{array}{lll}2.4 & \text { Colour of several particles } & 10\end{array}$

3 Impulse $\quad 11$

3.1 Colourful scattering observables in quantum field theory 11

$\begin{array}{ll}3.2 \text { Leading order } & 14\end{array}$

$\begin{array}{lll}3.3 & \text { Next to leading order } & 15\end{array}$

$\begin{array}{ll}3.3 .1 & \text { 1-loop amplitude } \\ 3.3 .2 & \text { Cht box }\end{array}$

$\begin{array}{lll}3.3 .2 & \text { Cut box } & 18\end{array}$

$\begin{array}{lll}3.3 .3 & \text { Combining terms } & 18\end{array}$

$\begin{array}{lll}3.3 .4 & \text { Final result } & 19\end{array}$

3.4 Momentum 20

4 Radiation 22

4.1 Total radiated colour 22

4.2 Leading order evaluation 24

5 Classical perspectives $\quad 26$

5.1 Impulses from equations of motion 26

$\begin{array}{lll}5.2 & \text { Total radiated colour and asymptotic symmetries } & 29\end{array}$

6 Discussion 31

A Charge factorisation in SU(3) 33

B Diagrams and amplitude expressions $\quad 34$

$\begin{array}{ll}\text { C Colour deflection for } N \text { particles } & 37\end{array}$

\section{Introduction}

Future prospects in gravitational wave astronomy require perturbative calculations at very high precision [1]. Remarkably, ideas and methods from quantum field theory offer a 
promising avenue of investigation [2-17]. Powerful techniques have been developed to compute scattering amplitudes, and we have learned how to extract classical physics efficiently from amplitudes [18-51]. A crucial insight from quantum field theory is the double copy: the observation that scattering amplitudes in gravitational theories can be computed from amplitudes in gauge theories. ${ }^{1}$ Since perturbation theory is far simpler in Yang-Mills (YM) theory than in standard approaches to gravity, the double copy has revolutionary potential.

Yang-Mills theory, treated as a classical field theory, shares many of the important physical features of gravity, including non-linearity and a subtle gauge structure. In this respect the YM case has always served as an excellent toy model for gravitational dynamics. But our developing understanding of the double copy has taught us that the connection between Yang-Mills theory and gravity is deeper than this. Detailed aspects of the perturbative dynamics of gravity, including gravitational radiation, can be deduced from Yang-Mills theory and the double copy $[11,21,53,54]$. In fact the double copy extends beyond perturbation theory, leading to exact maps [8, 55-81] between classical solutions of gauge theory and gravity, even when there is gravitational radiation present [9].

So we are motivated to take another look at perturbative YM processes, particularly those that connect to gravitational wave physics. However, the double copy is best understood as a relationship between the scattering amplitudes of YM theory and gravity. Consequently it is useful to formulate classical YM dynamics in terms of amplitudes, rather than by solving the equations of motion. We explain how to do so in this article. Since our interest is in the classical theory, we systematically ignore non-perturbative quantum effects. Nevertheless we identify observables which are well-defined in the classical theory and which can be extracted unambiguously from perturbative scattering amplitudes to any order. Our approach is complementary to an effort to understand the double copy from a purely classical, worldline perspective [11, 54, 82-89]. Perhaps the simplest way of implementing the double copy for classical quantities will be to compute observables using classical worldline methods; extract the relevant amplitudes by comparing to our formulae for observables in terms of amplitudes, and then double copy to gravity.

Returning briefly to our underlying interest in gravitational dynamics, it is worth emphasising the importance of spin. The spin of the individual bodies in a compact binary coalescence event influences the details of the outgoing gravitational radiation. This spin also contains information on the poorly-understood formation channels of the binaries. Measurement of spin is therefore one of the primary physics outputs of gravitational wave observations. It is fortunate, then, that spin can be incorporated rather naturally in the formalism of quantum field theory [41, 76, 90-104], even for very large (classical) spins. However the details of spin dynamics quickly becomes complex.

In the Yang-Mills context we can also discuss the dynamics of spin. However YangMills theories always involve colour, and colour is in many respects very analogous to spin. The dynamics of colour is actually a little simpler than spin, because the latter is tied together with spacetime while colour is defined in its own vector space. We therefore pay particular attention to the dynamics of colour in Yang-Mills theory. We discuss in detail

\footnotetext{
${ }^{1}$ The double copy was thoroughly reviewed recently [52].
} 
the change of colour of a particle during a scattering event, and the radiation of colour to null infinity. We also discuss more briefly the impulse (change of momentum) and radiation of momentum in YM theory. The methods we use build on ideas previously described by Kosower and two of us in a previous paper [15] (KMOC).

The classical equations of central interest in our paper describe the Yang-Mills field with some gauge group coupled to several classical point-like particles. These particles each carry colour charges $c^{a}$ which are time-dependent vectors in the adjoint representation of the gauge group. Often known as Wong's equations [105], the equations of motion for $N$ of these particles following worldlines $x_{\alpha}\left(\tau_{\alpha}\right)$ and with velocities $v_{\alpha}$ and momenta $p_{\alpha}$ are

$$
\begin{aligned}
\frac{\mathrm{d} p_{\alpha}^{\mu}}{\mathrm{d} \tau_{\alpha}} & =g c_{\alpha}^{a}\left(\tau_{\alpha}\right) F^{a \mu \nu}\left(x_{\alpha}\left(\tau_{\alpha}\right)\right) v_{\alpha \nu}\left(\tau_{\alpha}\right), \\
\frac{\mathrm{d} c_{\alpha}^{a}}{\mathrm{~d} \tau_{\alpha}} & =g f^{a b c} v_{\alpha}^{\mu}\left(\tau_{\alpha}\right) A_{\mu}^{b}\left(x_{\alpha}\left(\tau_{\alpha}\right)\right) c_{\alpha}^{c}\left(\tau_{\alpha}\right), \\
D^{\mu} F_{\mu \nu}^{a}(x)=J_{\nu}^{a}(x) & =g \sum_{\alpha=1}^{N} \int \mathrm{d} \tau_{\alpha} c_{\alpha}^{a}\left(\tau_{\alpha}\right) v_{\alpha \nu}\left(\tau_{\alpha}\right) \delta^{(4)}\left(x-x_{\alpha}\left(\tau_{\alpha}\right)\right),
\end{aligned}
$$

where the Yang-Mills field is $A_{\mu}=A_{\mu}^{a} T^{a}$ and $F_{\mu \nu}^{a}$ is the associated field strength tensor.

Although our motivation lies in gravitational dynamics, classical YM theory is also important in other contexts. Its asymptotic symmetry group has recently received intense study both for its own intrinsic interest but also as a toy model for the asymptotic symmetry group of gravity [106-112]. The radiation of colour charge of interest to us is also important in that context.

A totally different application is to the quark-gluon plasma, a high-temperature phase of matter which is described by the classical equations of Yang-Mills theory. Indeed it is in this context that the classical theory has its main application, as a tool to model transport phenomena in non-Abelian plasmas in the very high temperature regime [113118]. The equations of motion also provide a successful approximation for calculating the gluon distribution functions from deeply inelastic, ultrarelativistic ion collisions [119-121], and radiation from classical particles has recently been calculated in this regime $[122,123]$.

Our paper is organised as follows. In section 2 we review the theoretical treatment of colour in Yang-Mills theory, emphasising the importance of coherent states in the classical limit. We will see that point-like particles emerging from an underlying matter field in a representation $R$ of the gauge group have an adjoint-valued colour charge which naturally has dimensions of angular momentum. In section 3 we will set up expressions for the classical and momentum impulse when scattering states have colour, and provide an explicit computation of these observables at NLO. In section 4 we turn to radiation, constructing the total radiated colour charge in terms of amplitudes and explicitly recovering the classical LO radiation current derived in [11]. In section 5 we reproduce our earlier results using purely classical methods. Finally, we discuss our results in section 6 .

\section{Review of the theory of colour}

In this section we review the emergence of non-Abelian colour charges $c_{\alpha}^{a}-$ a vector in the adjoint representation for each particle species $\alpha$ - from a quantum field theory with 
scalars $\varphi_{\alpha}$ in any representation $R_{\alpha}$ of the gauge group, coupled to the Yang-Mills field. We specialise to the case of two different scalar fields, so that $\alpha=1,2$. Our action is

$$
S=\int \mathrm{d}^{4} x\left(\sum_{\alpha=1}^{2}\left[\left(D_{\mu} \varphi_{\alpha}^{\dagger}\right) D^{\mu} \varphi_{\alpha}-\frac{m_{\alpha}^{2}}{\hbar^{2}} \varphi_{\alpha}^{\dagger} \varphi_{\alpha}\right]-\frac{1}{4} F_{\mu \nu}^{a} F^{a \mu \nu}\right),
$$

where $D_{\mu}=\partial_{\mu}+i g A_{\mu}^{a} T_{R}^{a}$. We have restored factors of $\hbar$ for convenience below. The generator matrices (in a representation $R$ ) are $T_{R}^{a}=\left(T_{R}^{a}\right)_{i}{ }^{j}$ and satisfy the Lie algebra $\left[T_{R}^{a}, T_{R}^{b}\right]_{i}{ }^{j}=i f^{a b c}\left(T_{R}^{c}\right)_{i}{ }^{j}$. We take the metric signature to be mostly negative.

\subsection{Colour in classical field theory}

For simplicity we begin with the case of just a single massive scalar. At the classical level, the colour charge can be obtained from the Noether current $j_{\mu}^{a}$ associated with the global part of the gauge symmetry. The colour charge is explicitly given by

$$
\int \mathrm{d}^{3} x j_{0}^{a}(t, \boldsymbol{x})=i \int \mathrm{d}^{3} x\left(\varphi^{\dagger} T_{R}^{a} \partial_{0} \varphi-\left(\partial_{0} \varphi^{\dagger}\right) T_{R}^{a} \varphi\right) .
$$

Notice that a direct application of the Noether procedure has led to a colour charge with dimensions of action, or equivalently, of angular momentum.

It's worth dwelling on dimensional analysis in the context of the Wong equations (1.1) since they motivate us to make certain choices which may, at first, seem surprising. The Yang-Mills field strength

$$
F_{\mu \nu}^{a}=\partial_{\mu} A_{\nu}^{a}-\partial_{\nu} A_{\mu}^{a}-g f^{a b c} A_{\mu}^{b} A_{\nu}^{c}
$$

is obviously an important actor in these classical equations. Classical equations should contain no factors ${ }^{2}$ of $\hbar$, so we choose to maintain this precise expression for the field strength when $\hbar \neq 1$. By inspection it follows ${ }^{3}$ that $\left[g A_{\mu}^{a}\right]=L^{-1}$. We can develop this further; since the action of equation (2.1) has dimensions of angular momentum, the YangMills field strength must have dimensions of $\sqrt{M / L^{3}}$. Thus, from equation (2.3),

$$
\left[A_{\mu}^{a}\right]=\sqrt{\frac{M}{L}}, \quad[g]=\frac{1}{\sqrt{M L}} .
$$

This conclusion about the dimensions of $g$ is in contrast to the situation in electrodynamics, where $[e]=\sqrt{M L}$. Put another way, in electrodynamics the dimensionless fine structure constant is $e^{2} / 4 \pi \hbar$ while in our conventions the analogue is $\hbar g^{2} / 4 \pi$ ! It is possible to arrange matters such that the YM and EM cases are more similar, but we find the present conventions to be convenient in perturbative calculations.

Continuing with our discussion of dimensions, note that the Yang-Mills version of the Lorentz force, equation (1.1a), demonstrates that the quantity $g c^{a}$ must have the same

\footnotetext{
${ }^{2}$ An equivalent point of view is that any factors of $\hbar$ appearing in an equation which has classical meaning should be absorbed into parameters of the classical theory.

${ }^{3}$ We use the notation $[x]$ for the dimensions of the quantity $x$. The symbols $L$ and $M$ stand for dimension of length and mass, respectively.
} 
dimension as the electric charge. This is consistent with our observation above that the colour has dimensions of angular momentum.

At first our assignment of dimensions of $g$ may seem troubling from the perspective of extracting classical physics from scattering amplitudes following the algorithm described by Kosower and some of the authors (KMOC) [15]. The fact that $g$ has dimensions of $1 / \sqrt{M L}$ implies that the dimensionless coupling at each vertex is $g \sqrt{\hbar}$, so factors of $\hbar$ associated with the coupling appear with the opposite power to the case of electrodynamics (and gravity). However, because the colour charges are dimensionful the net power of $\hbar$ turns out to be the same, and thus the KMOC approach is ultimately unaltered. We will also see below that the dimensionful nature of the colour clarifies the classical limit of this aspect of the theory. To see how this works we must quantise.

\subsection{Colour of single-particle quantum states}

Dimensional analysis demonstrates that $\varphi$ has dimensions of $\sqrt{M / L}$, so its mode expansion is

$$
\varphi_{i}(x)=\frac{1}{\sqrt{\hbar}} \int \mathrm{d} \Phi(p)\left(a_{i}(p) e^{-i p \cdot x / \hbar}+b_{i}^{\dagger}(p) e^{i p \cdot x / \hbar}\right) .
$$

The index $i$ labels the representation $R$. We have normalised the ladder operators by requiring

$$
\left[a_{i}(p), a^{\dagger j}(q)\right]=2 E_{p}(2 \pi)^{3} \delta^{(3)}(\boldsymbol{p}-\boldsymbol{q}) \delta_{i}{ }^{j} \equiv \hat{\delta}_{\Phi}^{3}(p-q) \delta_{i}{ }^{j},
$$

and likewise for the antiparticle operators. Each ladder operator therefore has dimensions of $M^{-1}$. We also write the Lorentz-invariant phase space measure as

$$
\mathrm{d} \Phi(k)=\frac{\mathrm{d}^{4} k}{(2 \pi)^{4}} 2 \pi \Theta\left(k^{0}\right) \delta\left(k^{2}-m^{2}\right) \equiv \hat{\mathrm{d}}^{4} k \hat{\delta}^{(+)}\left(k^{2}-m^{2}\right),
$$

and introduce the hat notation in the measure and the delta functions

$$
\hat{\mathrm{d}}^{n} p \equiv \frac{\mathrm{d}^{n} p}{(2 \pi)^{n}}, \quad \hat{\delta}^{(n)}(x) \equiv(2 \pi)^{n} \delta^{(n)}(x)
$$

in order to avoid a proliferation of factors of $2 \pi$.

After quantisation, the colour charge of equation (2.2) becomes a Hilbert space operator which will be important below. To emphasise that this is an operator we write the quantised form as $\mathbb{C}^{a}$ :

$$
\begin{aligned}
\mathbb{C}^{a} & =i \int \mathrm{d}^{3} x\left(\varphi^{\dagger} T_{R}^{a} \partial_{0} \varphi-\left(\partial_{0} \varphi^{\dagger}\right) T_{R}^{a} \varphi\right) \\
& =\hbar \int \mathrm{d} \Phi(p)\left(a^{\dagger}(p) T_{R}^{a} a(p)+b^{\dagger}(p) T_{\bar{R}}^{a} b(p)\right),
\end{aligned}
$$

using the generators of the conjugate representation $\bar{R}, T_{\bar{R}}^{a}=-T_{R}^{a}$. The overall $\hbar$ factor guarantees that the colour has dimensions of angular momentum, as we require. It is important to note that these global colour operators inherit the usual Lie algebra of the generators, modified by factors of $\hbar$, so that

$$
\left[\mathbb{C}^{a}, \mathbb{C}^{b}\right]=i \hbar f^{a b c} \mathbb{C}^{c}
$$


Now we turn to the action of the colour operator on the single-particle states

$$
\left|p^{i}\right\rangle=a^{\dagger i}(p)|0\rangle
$$

Acting with the colour charge operator of equation (2.9) we immediately see that

$$
\mathbb{C}^{a}\left|p^{i}\right\rangle=\hbar\left(T_{R}^{a}\right)_{j}{ }^{i}\left|p^{j}\right\rangle, \quad\left\langle p_{i}\right| \mathbb{C}^{a}=\hbar\left\langle p_{j}\right|\left(T_{R}^{a}\right)_{i}{ }^{j}
$$

Thus inner products yield generators scaled by $\hbar$ :

$$
\left\langle p_{i}\left|\mathbb{C}^{a}\right| p^{j}\right\rangle \equiv\left(C^{a}\right)_{i}{ }^{j}=\hbar\left(T_{R}^{a}\right)_{i}{ }^{j}
$$

The $\left(C^{a}\right)_{i}{ }^{j}$ are simply rescalings of the usual generators $T_{R}^{a}$ by a factor of $\hbar$, and thus satisfy the rescaled Lie algebra in equation (2.10); since this rescaling is important for us, it is useful to make the distinction between the two.

We may then write a generic single particle state as

$$
|\psi\rangle=\sum_{i} \int \mathrm{d} \Phi(p) \phi(p) \chi_{i}\left|p^{i}\right\rangle,
$$

where the vector $\chi_{i}$ labels a general colour state, and the normalisations are chosen such that

$$
\int \mathrm{d} \Phi(p)|\phi(p)|^{2}=1, \quad \sum_{i} \chi^{i *} \chi_{i}=1
$$

The colour operator acts on these states as

$$
\mathbb{C}^{a}|\psi\rangle=\int \mathrm{d} \Phi(p)\left(C^{a}\right)_{i}^{j} \phi(p) \chi_{j}\left|p^{i}\right\rangle .
$$

Furthermore, we define the colour charge of the particle as

$$
\left\langle\psi\left|\mathbb{C}^{a}\right| \psi\right\rangle=\chi^{i *}\left(C^{a}\right)_{i}^{j} \chi_{j}
$$

Computing this charge and extracting its classical limit is the topic of the next section 2.3.

As a final remark on these rescaled generators, let us write out the covariant derivative in the representation $R$. In terms of $C^{a}$, the $\hbar$ scaling of interactions is precisely the same as in QED (and in perturbative gravity):

$$
D_{\mu}=\partial_{\mu}+i g A_{\mu}^{a} T^{a}=\partial_{\mu}+\frac{i g}{\hbar} A_{\mu}^{a} C^{a}
$$

for comparison, the covariant derivative used by KMOC in QED was $\partial_{\mu}+i e A_{\mu} / \hbar[15]$. Thus we have arranged that factors of $\hbar$ appear in the same place in YM theory as in electrodynamics, provided that the colour is measured by $C^{a}$. This ensures that the basic rules for obtaining the classical limits of amplitudes will be the same as in KMOC [15]. In practical calculations one can thus restore $\hbar$ 's in colour factors and work using $C^{a}$ 's everywhere. However, it is worth emphasising that unlike classical colour charges, the factors $C^{a}$ do not commute. 


\subsection{Colour of point-like particles in the classical regime}

The classical point-particle picture emerges from sharply peaked quantum wavepackets. In [15], linear exponential generalisations of Gaussian wavepackets were chosen for relativistic momentum space wavepackets. The essential property that must be satisfied was for the particle to have a sharply-defined position and a sharply-defined momentum whenever the classical limit was taken. To understand colour, governed by the Yang-Mills-Wong equations in the classical arena, a similar picture should emerge for our quantum colour operator in equation (2.9). Following the KMOC philosophy [15] we will first consider the full quantum states and then the classical limit. We define the classical limit of the colour charge to be

$$
c^{a} \equiv\left\langle\psi\left|\mathbb{C}^{a}\right| \psi\right\rangle .
$$

Our focus in this section is on the colour structure of our particle. The full state is a tensor product of colour and kinematics:

$$
|\psi\rangle=\sum\left|\psi_{\text {colour }}\right\rangle \otimes\left|\psi_{\text {kin }}\right\rangle,
$$

but as we ignore kinematics for now, we simply write the colour part of the state $\left|\psi_{\text {colour }}\right\rangle \rightarrow$ $|\psi\rangle$ in the remainder of this section. Then, in the classical limit, the critical requirements on the colour part of the state are that

$$
\begin{aligned}
\left\langle\psi\left|\mathbb{C}^{a}\right| \psi\right\rangle & =\text { finite } \\
\left\langle\psi\left|\mathbb{C}^{a} \mathbb{C}^{b}\right| \psi\right\rangle & =\left\langle\psi\left|\mathbb{C}^{a}\right| \psi\right\rangle\left\langle\psi\left|\mathbb{C}^{b}\right| \psi\right\rangle+\text { negligible }
\end{aligned}
$$

Since the colour operator explicitly involves a factor of $\hbar$, another parameter must be large so that the colour expectation $\left\langle\psi\left|\mathbb{C}^{a}\right| \psi\right\rangle$ is much bigger than $\hbar$ in the classical region. This situation is basically the same as for the usual classical limit of angular momentum: in that case we take the spin quantum number $j$ large so that $\hbar j$ is a classical angular momentum. For colour, we similarly need the size of the representation $R$ to be large. (We will see this explicitly in the case of SU(3) in a moment.) For the second requirement we must select appropriate colour wavefunctions $|\psi\rangle$ which are analogous to the kinematic wavepacket in KMOC [15].

Coherent states are the key to the classical limit very generally [124], including the case of angular momentum, so we choose a coherent state to describe the colour of our particle. The states used in KMOC to describe momenta [15] can themselves be understood as coherent states for a "first-quantised" particle - more specifically they are states for the restricted Poincaré group [125-127]. However, not all definitions of coherent states are equivalent, so we need to specify in what sense our states are coherent. The definition we use was introduced by Perelomov [128], which formalises the notion of coherent state for any Lie group and hence can be utilised for both the kinematic and the colour parts. It is in this sense that the states used by KMOC are coherent for the Poincaré group. We refer the interested reader to [129] for details of the Perelomov formalism and to [130] for applications.

For the explicit construction of the appropriate colour states we will use the Schwinger boson formalism. For $\mathrm{SU}(2)$, constructing irreducible representations from Schwinger 
bosons is a standard textbook exercise [131]. One simply introduces the Schwinger bosons - that is, creation $a^{\dagger i}$ and annihilation $a_{i}$ operators, transforming in the fundamental two-dimensional representation so that $i=1,2$. The irreducible representations of $\mathrm{SU}(2)$ are all symmetrised tensor powers of the fundamental, so the state

$$
a^{\dagger i_{1}} a^{\dagger i_{2}} \cdots a^{\dagger i_{2 j}}|0\rangle,
$$

which is automatically symmetric in all its indices, transforms in the spin $j$ representation.

For groups larger than $\mathrm{SU}(2)$, the situation is a little more complicated because the construction of a general irreducible representation requires both symmetrisation and antisymmetrisation over appropriate sets of indices. This leads to expressions which are involved already for $\mathrm{SU}(3)[132,133]$. We content ourselves with a brief discussion of the $\mathrm{SU}(3)$ case which captures all of the interesting features of the general case.

One can construct all irreducible representations from tensor products only of fundamentals $[134,135]$; however, for our treatment of $\mathrm{SU}(3)$ it is helpful to instead make use of the fundamental and antifundamental, and tensor these together to generate representations. Following [132], we introduce two sets of ladder operators $a_{i}$ and $b^{i}, i=1,2,3$, which transform in the $\mathbf{3}$ and $\mathbf{3}^{*}$ respectively. The colour operator can then be written as

$$
\mathbb{C}^{e}=\hbar\left(a^{\dagger} \frac{\lambda^{e}}{2} a-b^{\dagger} \frac{\bar{\lambda}^{e}}{2} b\right), \quad e=1, \ldots, 8
$$

where $\lambda^{e}$ are the Gell-Mann matrices and $\bar{\lambda}^{e}$ are their conjugates. The operators $a$ and $b$ satisfy the usual commutation relations

$$
\left[a_{i}, a^{\dagger j}\right]=\delta_{i}{ }^{j}, \quad\left[b^{i}, b_{j}^{\dagger}\right]=\delta^{i}{ }_{j}, \quad\left[a_{i}, b^{j}\right]=0, \quad\left[a^{\dagger i}, b_{j}^{\dagger}\right]=0 .
$$

By virtue of these commutators, the colour operator (2.23) obeys the commutation relation (2.10).

There are two Casimir operators given by the number operators ${ }^{4}$

$$
\mathcal{N}_{1} \equiv a^{\dagger} \cdot a, \quad \mathcal{N}_{2} \equiv b^{\dagger} \cdot b,
$$

with eigenvalues $n_{1}$ and $n_{2}$ respectively, so we label irreducible representations by $\left[n_{1}, n_{2}\right]$. Naïvely, the states we are looking for are constructed by acting on the vacuum state as follows:

$$
\left(a^{\dagger i_{1}} \cdots a^{\dagger i_{n_{1}}}\right)\left(b_{j_{1}}^{\dagger} \cdots b_{j_{n_{2}}}^{\dagger}\right)|0\rangle .
$$

However, these states are $\mathrm{SU}(3)$ reducible and thus cannot be used in our construction of coherent states. We write the irreducible states schematically by acting with a Young projector $\mathcal{P}$ which appropriately (anti-) symmetrises upper and lower indices, thereby subtracting traces:

$$
|\psi\rangle_{\left[n_{1}, n_{2}\right]} \equiv \mathcal{P}\left(\left(a^{\dagger i_{1}} \cdots a^{\dagger i_{n_{1}}}\right)\left(b_{j_{1}}^{\dagger} \cdots b_{j_{n_{2}}}^{\dagger}\right)|0\rangle\right) .
$$

In general these operations will lead to involved expressions for the states, but we can understand them from their associated Young tableaux (figure 1). Each double box column 


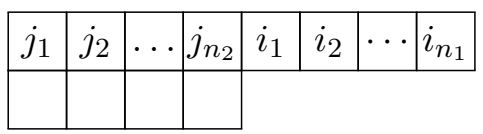

Figure 1. Young tableau of $\mathrm{SU}(3)$.

represents an operator $b_{i}^{\dagger}$ and each single column box represents the operator $a^{\dagger i}$, and thus for a mixed representation we have $n_{2}$ double columns and $n_{1}$ single columns.

Having constructed the irreducible states, one can define a coherent state parametrised by two triplets of complex numbers $\xi_{i}$ and $\zeta^{i}, i=1,2,3$. These are normalised according to

$$
|\xi|^{2}=|\zeta|^{2}=1, \quad \xi \cdot \zeta=0
$$

We won't require fully general coherent states, but instead their projections onto the $\left[n_{1}, n_{2}\right]$ representation, which are

$$
|\xi \zeta\rangle_{\left[n_{1}, n_{2}\right]} \equiv \frac{1}{\sqrt{\left(n_{1} ! n_{2} !\right)}}\left(\zeta \cdot b^{\dagger}\right)^{n_{2}}\left(\xi \cdot a^{\dagger}\right)^{n_{1}}|0\rangle .
$$

The square roots ensure that the states are normalised to unity. ${ }^{5}$ With this normalisation we can write the identity operator as

$$
\mathbb{1}_{\left[n_{1}, n_{2}\right]}=\int \mathrm{d} \mu(\xi, \zeta)(|\xi \zeta\rangle\langle\xi \zeta|)_{\left[n_{1}, n_{2}\right]},
$$

where $\int \mathrm{d} \mu(\xi, \zeta)$ is the $\mathrm{SU}(3)$ Haar measure, normalised such that $\int \mathrm{d} \mu(\xi, \zeta)=1$. Its precise form is irrelevant for our purposes.

With the states in hand, we can return to the expectation value of the colour operator $\mathbb{C}^{a}$ in equation (2.9). The size of the representation, that is $n_{1}$ and $n_{2}$, must be large compared to $\hbar$ in the classical regime so that the final result is finite. To see this let us compute this expectation value explicitly. By definition we have

$$
\left\langle\xi \zeta\left|\mathbb{C}^{e}\right| \xi \zeta\right\rangle_{\left[n_{1}, n_{2}\right]}=\frac{\hbar}{2}\left(\left\langle\xi \zeta\left|a^{\dagger} \lambda^{e} a\right| \xi \zeta\right\rangle_{\left[n_{1}, n_{2}\right]}-\left\langle\xi \zeta\left|b^{\dagger} \bar{\lambda}^{e} b\right| \xi \zeta\right\rangle_{\left[n_{1}, n_{2}\right]}\right) .
$$

After a little algebra we find that

$$
\left\langle\xi \zeta\left|\mathbb{C}^{e}\right| \xi \zeta\right\rangle=\frac{\hbar}{2}\left(n_{1} \xi^{*} \lambda^{e} \xi-n_{2} \zeta^{*} \bar{\lambda}^{e} \zeta\right)
$$

We see that a finite charge requires a scaling limit in which we take $n_{1}, n_{2}$ large as $\hbar \rightarrow 0$, keeping the product $\hbar n_{i}$ fixed for at least one value of $i$. The classical charge is therefore the finite c-number

$$
c^{a}=\left\langle\xi \zeta\left|\mathbb{C}^{a}\right| \xi \zeta\right\rangle_{\left[n_{1}, n_{2}\right]}=\frac{\hbar}{2}\left(n_{1} \xi^{*} \lambda^{a} \xi-n_{2} \zeta^{*} \bar{\lambda}^{a} \zeta\right) .
$$

\footnotetext{
${ }^{4}$ Here we define $a^{\dagger} \cdot a \equiv \sum_{i=1}^{3} a^{\dagger i} a_{i}$ and $|\xi|^{2} \equiv \sum_{i=1}^{3}\left|\xi_{i}\right|^{2}$.

${ }^{5}$ Note that the Young projector in equation $(2.27)$ is no longer necessary since the constraint $\xi \cdot \zeta=0$ removes all the unwanted traces.
} 
The other feature we must check is the expectation value of products. A similar calculation for two pairs of charge operators in a large representation leads to the important property

$$
\begin{aligned}
\left\langle\xi \zeta\left|\mathbb{C}^{a} \mathbb{C}^{b}\right| \xi \zeta\right\rangle_{\left[n_{1}, n_{2}\right]} & =\left\langle\xi \zeta\left|\mathbb{C}^{a}\right| \xi \zeta\right\rangle_{\left[n_{1}, n_{2}\right]}\left\langle\xi \zeta\left|\mathbb{C}^{b}\right| \xi \zeta\right\rangle_{\left[n_{1}, n_{2}\right]}+\mathcal{O}(\hbar) \\
& =c^{a} c^{b}+\mathcal{O}(\hbar) .
\end{aligned}
$$

This is in fact a special case of a more general construction discussed in detail by Yaffe [124]. In appendix A we prove equation (2.34), and show that the correction term is $\mathcal{O}(\hbar)$. The same argument can be used to demonstrate an important property of the coherent states in the classical limit, which is that the overlap $\left\langle\chi^{\prime} \mid \chi\right\rangle$ is very strongly peaked about $\chi=$ $\chi^{\prime}$ [124]. We have thus constructed explicit colour states which ensure the correct classical behaviour of the colour charges.

In the calculation of the colour impulse and radiated colour in sections 3 and 4 , we will only need to make use of the finiteness and factorisation properties, so we will avoid further use of the explicit form of the states. Henceforth we write $\chi_{i}$ for the parameters of a general colour state $|\chi\rangle$ with these properties, and $\mathrm{d} \mu(\chi)$ for the Haar measure of the colour group, whatever it may be.

\subsection{Colour of several particles}

Now that we have reviewed the theory of colour for a single particle, it's time to consider what happens when more than one particle is present. We will shortly discuss the dynamics of colour in detail; here, we set up initial states describing more than one point-like particle.

We take our particles to be distinguishable, so they are associated with distinct quantum fields $\varphi_{\alpha}$ with $\alpha=1,2$. We only consider two different particles explicitly, though it is no more difficult to consider the many-particle case. We also restrict to scalar fields, again for simplicity. The action is therefore as given in equation (2.1). Both fields $\varphi_{\alpha}$ must be in representations $R_{\alpha}$ which are large, so that a classical limit is available for the individual colours.

At some initial time in the far past, we assume that our two particles both have welldefined positions, momenta and colours. In other words, particle $\alpha$ has a wavepacket $\phi_{\alpha}\left(p_{\alpha}\right)$ describing its momentum-space distribution, and a colour wavepacket $\chi_{\alpha}$ as described in section 2.3. In this initial state, both particles are separated by an impact parameter $b$ (which must then be very large compared to the spatial spread of the momenta in the wavepackets [15]). We further assume that there is no incoming radiation (that is, no vector boson) in the incoming state. Thus, the state is

$$
\begin{aligned}
|\Psi\rangle & =\int \mathrm{d} \Phi\left(p_{1}\right) \mathrm{d} \Phi\left(p_{2}\right) \phi_{1}\left(p_{1}\right) \phi_{2}\left(p_{2}\right) e^{i b \cdot p_{1} / \hbar}\left|p_{1} \chi_{1} ; p_{2} \chi_{2}\right\rangle \\
& =\int \mathrm{d} \Phi\left(p_{1}\right) \mathrm{d} \Phi\left(p_{2}\right) \phi_{1}\left(p_{1}\right) \phi_{2}\left(p_{2}\right) e^{i b \cdot p_{1} / \hbar} \chi_{1 i} \chi_{2 j}\left|p_{1}{ }^{i} ; p_{2}{ }^{j}\right\rangle .
\end{aligned}
$$

Notice that the state $|\Psi\rangle$ refers to a multi-particle state. We reserve the notation $|\psi\rangle$ for single particle states.

We measure the colour of multi-particle states by acting with a colour operator which is simply the sum of the individual colour operators (2.9) for each of the scalar fields. For 
example, acting on the state $\left|p_{1} \chi_{1} ; p_{2} \chi_{2}\right\rangle$ we have

$$
\begin{aligned}
\mathbb{C}^{a}\left|p_{1} \chi_{1} ; p_{2} \chi_{2}\right\rangle & =\left|p_{1}{ }^{i^{\prime}} p_{2}{ }^{j^{\prime}}\right\rangle\left(\left(C_{1}^{a}\right)_{i^{\prime}}{ }^{i} \delta_{j^{\prime}}{ }^{j}+\delta_{i^{\prime}}{ }^{i}\left(C_{2}^{a}\right)_{j^{\prime}}{ }^{j}\right) \chi_{1 i} \chi_{2 j} \\
& =\int \mathrm{d} \mu\left(\chi_{1}^{\prime}\right) \mathrm{d} \mu\left(\chi_{2}^{\prime}\right)\left|p_{1} \chi_{1}^{\prime} ; p_{2} \chi_{2}^{\prime}\right\rangle\left\langle\chi_{1}^{\prime} \chi_{2}^{\prime}\left|C_{1}^{a} \otimes 1+1 \otimes C_{2}^{a}\right| \chi_{1} \chi_{2}\right\rangle \\
& =\int \mathrm{d} \mu\left(\chi_{1}^{\prime}\right) \mathrm{d} \mu\left(\chi_{2}^{\prime}\right)\left|p_{1} \chi_{1}^{\prime} ; p_{2} \chi_{2}^{\prime}\right\rangle\left\langle\chi_{1}^{\prime} \chi_{2}^{\prime}\left|C_{1+2}^{a}\right| \chi_{1} \chi_{2}\right\rangle,
\end{aligned}
$$

where $C_{\alpha}^{a}$ is the colour in representation $R_{\alpha}$ and we have written $C_{1+2}^{a}$ for the colour operator on the tensor product of representations $R_{1}$ and $R_{2}$. In the classical regime, using the property that the overlap between states sets $\chi_{i}^{\prime}=\chi_{i}$ in the classical limit, it follows that

$$
\left\langle p_{1} \chi_{1} ; p_{2} \chi_{2}\left|C_{1+2}^{a}\right| p_{1} \chi_{1} ; p_{2} \chi_{2}\right\rangle=c_{1}^{a}+c_{2}^{a},
$$

so the colours simply add.

\section{Impulse}

Now we move on to the dynamics of colour. Our focus in this section will be on the colour impulse - that is, the total change in colour during a scattering event - leaving radiation of colour to the next section. We begin by setting up the colour impulse observable in the vein of $[15,95]$ before turning to explicit examples at LO and NLO.

\subsection{Colourful scattering observables in quantum field theory}

A natural observable in Yang-Mills theory is the total change in the colour charge of one of the massive scattering particles,

$$
\begin{aligned}
\left\langle\Delta c_{1}^{a}\right\rangle & =\left\langle\Psi\left|S^{\dagger} \mathbb{C}_{1}^{a} S\right| \Psi\right\rangle-\left\langle\Psi\left|\mathbb{C}_{1}^{a}\right| \Psi\right\rangle \\
& =i\left\langle\Psi\left|\left[\mathbb{C}_{1}^{a}, T\right]\right| \Psi\right\rangle+\left\langle\Psi\left|T^{\dagger}\left[\mathbb{C}_{1}^{a}, T\right]\right| \Psi\right\rangle,
\end{aligned}
$$

where we have introduced the $S$ and $T$ matrices, related by $S=1+i T$, and utilised the optical theorem. We call this observable the colour impulse, as it mirrors the structure of the momentum impulse $\Delta p_{1}^{\mu}$ of [15] and angular impulse $\Delta s_{1}^{\mu}$ of [95]. An immediate novelty for this impulse is that it is a Lorentz scalar, instead transforming in the adjoint representation of the gauge group.

Substituting the 2-particle wavepackets in equation (2.35) yields

$$
\begin{aligned}
\left\langle\Delta c_{1}^{a}\right\rangle= & \prod_{i=1,2} \int \mathrm{d} \Phi\left(p_{i}\right) \mathrm{d} \Phi\left(p_{i}^{\prime}\right) \phi_{i}\left(p_{i}\right) \phi_{i}^{*}\left(p_{i}^{\prime}\right) e^{i b \cdot\left(p_{1}-p_{1}^{\prime}\right) / \hbar} \\
& \times\left\langle p_{1}^{\prime} \chi_{1} ; p_{2}^{\prime} \chi_{2}\left|i\left[\mathbb{C}_{1}^{a}, T\right]+T^{\dagger}\left[\mathbb{C}_{1}^{a}, T\right]\right| p_{1} \chi_{1} ; p_{2} \chi_{2}\right\rangle,
\end{aligned}
$$

which we expand in terms of amplitudes by inserting complete sets of states,

$$
\mathbb{1}=\sum_{X} \int \mathrm{d} \Phi\left(r_{1}\right) \mathrm{d} \Phi\left(r_{2}\right) \mathrm{d} \mu\left(\zeta_{1}\right) \mathrm{d} \mu\left(\zeta_{2}\right)\left|r_{1} \zeta_{1} ; r_{2} \zeta_{2} ; X\right\rangle\left\langle r_{1} \zeta_{1} ; r_{2} \zeta_{2} ; X\right|
$$

The set $X$ could contain any number of extra gluon or scalar states, whose phase space measures and sums over any other quantum numbers are left implicit in the summation over $X$. 
It is frequently convenient to write amplitudes in Yang-Mills theory in colour-ordered form; for example, see [136] for an application to amplitudes with multiple different external particles. The full amplitude $\mathcal{A}$ is decomposed onto a basis of colour factors times partial amplitudes $A$. The colour factors are associated with some set of Feynman topologies. Once a basis of independent colour structures is chosen, the corresponding partial amplitudes must be gauge invariant. Thus,

$$
\begin{aligned}
\left\langle p_{1}^{\prime} \chi_{1} ; p_{2}^{\prime} \chi_{2}|T| p_{1} \chi_{1} ; p_{2} \chi_{2}\right\rangle & =\left\langle\chi_{1} \chi_{2}\left|\mathcal{A}\left(p_{1}, p_{2} \rightarrow p_{1}^{\prime}, p_{2}^{\prime}\right)\right| \chi_{1} \chi_{2}\right\rangle \hat{\delta}^{(4)}\left(p_{1}+p_{2}-p_{1}^{\prime}-p_{2}^{\prime}\right) \\
& =\sum_{D}\langle\mathcal{C}(D)\rangle A_{D}\left(p_{1}, p_{2} \rightarrow p_{1}^{\prime}, p_{2}^{\prime}\right) \hat{\delta}^{(4)}\left(p_{1}+p_{2}-p_{1}^{\prime}-p_{2}^{\prime}\right)
\end{aligned}
$$

where $\mathcal{C}(D)$ is the colour factor of diagram $D$ and $A_{D}$ is the associated partial amplitude. The remaining expectation value is over the colour states $\chi_{i}$. Using this notation and the action of the colour operator in equation (2.36), we can write the colour impulse as

$$
\begin{aligned}
\left\langle\Delta c_{1}^{a}\right\rangle= & \prod_{i=1,2} \sum_{D} \int \mathrm{d} \Phi\left(p_{i}\right) \mathrm{d} \Phi\left(p_{i}^{\prime}\right) \phi_{i}\left(p_{i}\right) \phi_{i}^{*}\left(p_{i}^{\prime}\right) e^{i b \cdot\left(p_{1}-p_{1}^{\prime}\right) / \hbar} \\
\times & {\left[i\left\langle\left[C_{1}^{a}, \mathcal{C}(D)\right]\right\rangle A_{D}\left(p_{1}, p_{2} \rightarrow p_{1}^{\prime}, p_{2}^{\prime}\right) \hat{\delta}^{(4)}\left(p_{1}+p_{2}-p_{1}^{\prime}-p_{2}^{\prime}\right)\right.} \\
& +\sum_{D^{\prime}} \sum_{X} \int \mathrm{d} \Phi\left(r_{i}\right)\left\langle\mathcal{C}\left(D^{\prime}\right)^{\dagger}\left[C_{1}^{a}, \mathcal{C}(D)\right]\right\rangle A_{D^{\prime}}^{*}\left(p_{1}^{\prime}, p_{2}^{\prime} \rightarrow r_{1}, r_{2}, r_{X}\right) \\
& \left.\times A_{D}\left(p_{1}, p_{2} \rightarrow r_{1}, r_{2}, r_{X}\right) \hat{\delta}^{(4)}\left(p_{1}+p_{2}-p_{1}^{\prime}-p_{2}^{\prime}\right) \hat{\delta}^{(4)}\left(p_{1}+p_{2}-r_{1}-r_{2}-r_{X}\right)\right] .
\end{aligned}
$$

Finally, let us introduce the momentum mismatch $q_{i}=p_{i}^{\prime}-p_{i}$, and transfer $w_{i}=r_{i}-p_{i}$. After integrating over the delta functions, we arrive at

$$
\begin{aligned}
\left\langle\Delta c_{1}^{a}\right\rangle= & i \int \mathrm{d} \Phi\left(p_{1}\right) \mathrm{d} \Phi\left(p_{2}\right) \hat{\mathrm{d}}^{4} q \hat{\delta}\left(2 p_{1} \cdot q+q^{2}\right) \hat{\delta}\left(2 p_{2} \cdot q-q^{2}\right) \\
\times & \Theta\left(p_{1}^{0}+q^{0}\right) \Theta\left(p_{2}^{0}-q^{0}\right) \phi_{1}\left(p_{1}\right) \phi_{2}\left(p_{2}\right) \phi_{1}^{*}\left(p_{1}+q\right) \phi_{2}^{*}\left(p_{2}-q\right) e^{-i b \cdot q / \hbar} \\
\times & \left\{\sum_{D}\left\langle\left[C_{1}^{a}, \mathcal{C}(D)\right]\right\rangle A_{D}\left(p_{1}, p_{2} \rightarrow p_{1}+q, p_{2}-q\right)\right. \\
& \quad-i \prod_{i=1,2} \sum_{X} \int \hat{\mathrm{d}}^{4} w_{i} \hat{\delta}\left(2 p_{1} \cdot w_{i}+w_{i}^{2}\right) \hat{\delta}^{(4)}\left(w_{1}+w_{2}-r_{X}\right) \Theta\left(p_{i}^{0}+w_{i}^{0}\right) \\
& \times \sum_{D, D^{\prime}}\left\langle\mathcal{C}\left(D^{\prime}\right)^{\dagger}\left[C_{1}^{a}, \mathcal{C}(D)\right]\right\rangle A_{D}\left(p_{1}, p_{2} \rightarrow p_{1}+w_{1}, p_{2}+w_{2}, r_{X}\right) \\
& \left.\times A_{D^{\prime}}^{*}\left(p_{1}+q, p_{2}-q \rightarrow p_{1}+w_{1}, p_{2}+w_{2}, r_{X}\right)\right\} .
\end{aligned}
$$

It is interesting to note that factors of expectation values of colour commutators, such as $\left\langle\left[C_{1}^{a}, \mathcal{C}(D)\right]\right\rangle$, in the colour impulse play a similar role to that of the momentum mismatch $q^{\mu}$ in the momentum impulse $\Delta p_{1}^{\mu}[15]$.

The momentum impulse in QED and gravity was discussed in detail in [15]. In YangMills theory, the presence of colour leads to slight modifications of those KMOC expressions. The basic difference is the colour structure of the amplitude. The observable itself is built 
from the (colour singlet) momentum operator $\mathbb{P}_{1}^{\mu}$, so factors of $C_{1}^{a}$ appearing in the colour impulse, equation (3.6), do not arise in the momentum case. We proceed by writing the full amplitude as a sum over colour structures, finding

$$
\begin{aligned}
\left\langle\Delta p_{1}^{\mu}\right\rangle= & i \int \mathrm{d} \Phi\left(p_{1}\right) \mathrm{d} \Phi\left(p_{2}\right) \hat{\mathrm{d}}^{4} q \hat{\delta}\left(2 p_{1} \cdot q+q^{2}\right) \hat{\delta}\left(2 p_{2} \cdot q-q^{2}\right) \\
\times & \Theta\left(p_{1}^{0}+q^{0}\right) \Theta\left(p_{2}^{0}-q^{0}\right) \phi\left(p_{1}\right) \phi\left(p_{2}\right) \phi^{*}\left(p_{1}+q\right) \phi^{*}\left(p_{2}-q\right) e^{-i b \cdot q} \\
\times & \left\{\sum_{D} q^{\mu}\langle\mathcal{C}(D)\rangle A_{D}\left(p_{1}, p_{2} \rightarrow p_{1}+q, p_{2}-q\right)\right. \\
& -i \prod_{i=1,2} \sum_{X} \int \hat{\mathrm{d}}^{4} w_{i} \hat{\delta}\left(2 p_{1} \cdot w_{i}+w_{i}^{2}\right) \hat{\delta}^{(4)}\left(w_{1}+w_{2}-r_{X}\right) \Theta\left(p_{i}^{0}+w_{i}^{0}\right) \\
& \times \sum_{D, D^{\prime}}\left\langle\mathcal{C}\left(D^{\prime}\right)^{\dagger} \mathcal{C}(D)\right\rangle A_{D}\left(p_{1}, p_{2} \rightarrow p_{1}+w_{1}, p_{2}+w_{2}, r_{X}\right) \\
& \left.\times A_{D^{\prime}}^{*}\left(p_{1}+q, p_{2}-q \rightarrow p_{1}+w_{1}, p_{2}+w_{2}, r_{X}\right)\right\} .
\end{aligned}
$$

By construction, both impulse observables are well defined in the classical regime. Once wavefunctions of the types described in section 2 are used, the details of the wavefunctions will not be important. However, to extract expressions which are valid in the classical approximation, it is important to be aware that the commutators of the $C^{a}$ contain powers of $\hbar$. In particular one must take care to expand all commutators of colour factors.

All other powers of $\hbar$ appear as described by KMOC. In brief, the rescaled covariant derivative of equation (2.18) ensures that each factor of the coupling $g$ is accompanied by a factor $\hbar^{-1 / 2}$; all massless external and loop momenta are products of a factor of $\hbar$ and a wavenumber; care must be taken with squares of massless momenta $q^{2}$ in delta functions. Finally, small shifts of order $\hbar \bar{q}$ to the dominant momenta of order $m$ in wavefunctions can be neglected; this is an example of a general property of coherent states in the classical limit [124]. We therefore introduce the notation

$$
\left\langle\left\langle f\left(p_{1}, p_{2}, \cdots\right)\right\rangle\right\rangle=\int \mathrm{d} \Phi\left(p_{1}\right) \mathrm{d} \Phi\left(p_{2}\right)\left|\phi\left(p_{1}\right)\right|^{2}\left|\phi\left(p_{2}\right)\right|^{2}\left\langle\chi_{1} \chi_{2}\left|f\left(p_{1}, p_{2}, \cdots\right)\right| \chi_{1} \chi_{2}\right\rangle .
$$

The nature of the wavepackets make evaluating these expectation values very easy in the classical limit: the momentum phase space integrals are simply evaluated by replacing massive momenta with 4 -velocities, $p_{i} \rightarrow m_{i} u_{i}$ [15], while the colour expectation value is guaranteed by equation (2.34) to behave as a product of commuting classical colour charges. We will still use single angle brackets to indicate expectation values which are only over the colour states.

Following this procedure, the colour impulse becomes

$$
\left\langle\Delta c_{1}^{a}\right\rangle \rightarrow \Delta c_{1}^{a}=i\left\langle\left\langle\int \hat{\mathrm{d}}^{4} \bar{q} \hat{\delta}\left(2 p_{1} \cdot \bar{q}\right) \hat{\delta}\left(2 p_{2} \cdot \bar{q}\right) e^{-i b \cdot \bar{q}} \mathcal{G}^{a} 》\right\rangle\right.
$$


where we define the colour kernel $\mathcal{G}^{a}$ to be

$$
\begin{aligned}
& \mathcal{G}^{a}=\hbar^{2} \sum_{D}\left[C_{1}^{a}, \mathcal{C}(D)\right] A_{D}\left(p_{1}, p_{2} \rightarrow p_{1}+q, p_{2}-q\right) \\
& -i \hbar^{4} \sum_{X} \prod_{i=1,2} \int \hat{\mathrm{d}}^{4} \bar{w}_{i} \hat{\delta}\left(2 p_{1} \cdot \bar{w}_{i}+\hbar \bar{w}_{i}^{2}\right) \hat{\delta}^{(4)}\left(\bar{w}_{1}+\bar{w}_{2}-\bar{r}_{X}\right) \sum_{D, D^{\prime}} \mathcal{C}\left(D^{\prime}\right)^{\dagger}\left[C^{a}, \mathcal{C}(D)\right] \\
& \times A_{D^{\prime}}^{*}\left(p_{1}+q, p_{2}-q \rightarrow p_{1}+w_{1}, p_{2}+w_{2}, r_{X}\right) A_{D}\left(p_{1}, p_{2} \rightarrow p_{1}+w_{1}, p_{2}-w_{2}, r_{X}\right) .
\end{aligned}
$$

We designed this kernel so that it is of order $\hbar^{0}$ in the classical approximation. Clearly at LO only the first term, linear in the amplitude, contributes; the second integral contributes from NLO, where it is an integral over tree level diagrams, while the first term involves one-loop amplitudes.

In the same notation, the momentum impulse is

$$
\left\langle\Delta p_{1}^{\mu}\right\rangle \rightarrow \Delta p_{1}^{\mu}=i\left\langle\left\langle\int \hat{\mathrm{d}}^{4} \bar{q} \hat{\delta}\left(2 p_{1} \cdot \bar{q}\right) \hat{\delta}\left(2 p_{2} \cdot \bar{q}\right) e^{-i b \cdot \bar{q}} \mathcal{I}^{\mu} \rrbracket\right\rangle\right.
$$

with momentum kernel

$$
\begin{aligned}
\mathcal{I}^{\mu}= & \hbar^{3} \bar{q}^{\mu} \sum_{D} \mathcal{C}(D) A_{D}\left(p_{1}, p_{2} \rightarrow p_{1}+q, p_{2}-q\right) \\
& -i \hbar^{5} \sum_{X} \prod_{i=1,2} \int \hat{\mathrm{d}}^{4} \bar{w}_{i} \hat{\delta}\left(2 p_{i} \cdot \bar{w}_{i}+\hbar \bar{w}_{i}^{2}\right) \hat{\delta}^{(4)}\left(\bar{w}_{1}+\bar{w}_{2}-\bar{r}_{X}\right) \bar{w}_{1}^{\mu} \sum_{D, D^{\prime}} \mathcal{C}\left(D^{\prime}\right)^{\dagger} \mathcal{C}(D) \\
& \times A_{D^{\prime}}^{*}\left(p_{1}+q, p_{2}-q \rightarrow p_{1}+w_{1}, p_{2}+w_{2}, r_{X}\right) A_{D}\left(p_{1}, p_{2} \rightarrow p_{1}+w_{1}, p_{2}+w_{2}, r_{X}\right)
\end{aligned}
$$

when the scattering particles carry colour.

\subsection{Leading order}

We may now compute the colour impulse explicitly. We begin at leading order (LO) for the scalar YM theory defined by equation (2.1), moving to next-to-leading order (NLO) in the next subsection. We will strip coupling constants from amplitudes, writing $\bar{A}_{D}^{(n)}$ for the charge stripped partial amplitudes at $\mathcal{O}\left(g^{2 n+2}\right)$. At LO the colour kernel is

$$
\mathcal{G}^{a,(0)}=\hbar g^{2} \sum_{D}\left[C_{1}^{a}, \mathcal{C}(D)\right] \bar{A}_{D}^{(0)}\left(p_{1}, p_{2} \rightarrow p_{1}+\hbar \bar{q}, p_{2}-\hbar \bar{q}\right)
$$

Here only the $t$-channel tree topology contributes, so the sum between colour and kinematics is trivial; we simply have ${ }^{6}$

$$
\bar{A}_{\succ}=\frac{4 p_{1} \cdot p_{2}+\hbar \bar{q}^{2}}{\hbar^{2} \bar{q}^{2}}, \quad \mathcal{C}\left(〉\langle)=C_{1} \cdot C_{2}\right.
$$

and therefore the colour impulse factor is

$$
\left[C_{1}^{a}, \mathcal{C}(\succ \prec)\right]=\left[C_{1}^{a}, C_{1}^{b}\right] C_{2}^{b}=i \hbar f^{a b c} C_{1}^{c} C_{2}^{b} .
$$

\footnotetext{
${ }^{6}$ We adopt the convention that time runs vertically in Feynman diagrams.
} 
Inserting these expressions into the colour kernel, all factors of $\hbar$ cancel as expected for a classical observable. The classical limit is

$$
\begin{aligned}
\Delta c_{1}^{a,(0)} & =-g^{2}\left\langle\left\langle f^{a b c} C_{1}^{c} C_{2}^{b} \int \hat{\mathrm{d}}^{4} \bar{q} \hat{\delta}\left(p_{1} \cdot \bar{q}\right) \hat{\delta}\left(p_{2} \cdot \bar{q}\right) e^{-i b \cdot \bar{q}} \frac{p_{1} \cdot p_{2}}{\bar{q}^{2}}\right\rangle\right. \\
& =g^{2} f^{a b c} c_{1}^{b} c_{2}^{c} u_{1} \cdot u_{2} \int \hat{\mathrm{d}}^{4} \bar{q} \hat{\delta}\left(u_{1} \cdot \bar{q}\right) \hat{\delta}\left(u_{2} \cdot \bar{q}\right) \frac{e^{-i b \cdot \bar{q}}}{\bar{q}^{2}} .
\end{aligned}
$$

Notice that while evaluating the large double angle brackets we obtained classical colour charges as expectations values of the $C_{\alpha}$.

The remaining integral is straightforward but divergent. While we use dimensional regulation throughout the remainder of the paper to define divergent integrals, in this case it is convenient to take a different approach.

The logarithmic divergence in the colour may seem surprising at first. However, the spacetime position of the particle is also logarithmically divergent in four dimensions; this is simply the familiar divergence due to the long-range nature of $1 / r^{2}$ forces in four dimensions. We therefore introduce a cutoff regulator $L$ of dimensions length as follows. Consider the following quantity

$$
2 b^{2} \frac{\partial \Delta c_{1}^{a,(0)}}{\partial b^{2}}=b_{\mu} \frac{\partial \Delta c_{1}^{a,(0)}}{\partial b_{\mu}}=-i g^{2} f^{a b c} c_{1}^{b} c_{2}^{c} \gamma b_{\mu} \int \hat{\mathrm{d}}^{4} \bar{q} \hat{\delta}\left(u_{1} \cdot \bar{q}\right) \hat{\delta}\left(u_{2} \cdot \bar{q}\right) \frac{e^{-i b \cdot \bar{q}} \bar{q}^{\mu}}{\bar{q}^{2}}
$$

where $\gamma=u_{1} \cdot u_{2}$. The integral on the r.h.s. was evaluated in [15]. Using that result, it is easy to show that the solution of the differential equation is

$$
\Delta c_{1}^{a,(0)}=\frac{\gamma g^{2} f^{a b c} c_{1}^{b} c_{2}^{c}}{4 \pi \sqrt{\gamma^{2}-1}} \log \left(\frac{b^{2}}{L^{2}}\right)
$$

where we have included the regulator explicitly.

\subsection{Next to leading order}

At NLO the classical colour kernel, with $\hbar$ 's from couplings removed, is

$$
\begin{aligned}
\mathcal{G}^{a,(1)}= & g^{4} \sum_{\Gamma}\left[C_{1}^{a}, \mathcal{C}(\Gamma)\right] \bar{A}_{\Gamma}^{(1)}\left(p_{1}, p_{2} \rightarrow p_{1}+\hbar \bar{q}, p_{2}-\hbar \bar{q}\right) \\
& -i g^{4} \hbar^{2} \int \hat{\mathrm{d}}^{4} \bar{\ell} \hat{\delta}\left(2 p_{1} \cdot \bar{\ell}+\hbar \bar{\ell}^{2}\right) \hat{\delta}\left(2 p_{2} \cdot \bar{\ell}-\hbar \bar{\ell}^{2}\right) \mathcal{C}\left(\succ\langle)^{\dagger}\left[C_{1}^{a}, \mathcal{C}(\succ \measuredangle)\right]\right. \\
& \times \bar{A}_{\succ}^{*}\left(p_{1}+\hbar \bar{q}, p_{2}-\hbar \bar{q} \rightarrow p_{1}+\hbar \bar{\ell}, p_{2}-\hbar \bar{\ell}\right) \bar{A}_{\succ}\left(p_{1}, p_{2} \rightarrow p_{1}+\hbar \bar{\ell}, p_{2}-\hbar \bar{\ell}\right),
\end{aligned}
$$

where $\Gamma$ is a set of one-loop topologies which span the independent colour factors. By the analysis of [15], the topologies relevant in the classical regime are ${ }^{7}$

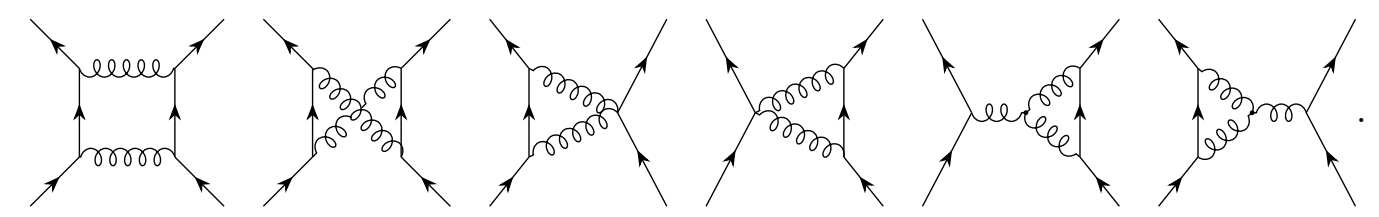

\footnotetext{
${ }^{7}$ See appendix B for the evaluation of these diagrams.
} 
We will refer to these as the box $B$, cross box $C$, triangles $T_{i j}$ and non-Abelian diagrams $Y_{i j}$ respectively. The latter, involving the 3 -gluon interaction vertex, are new to the YangMills calculation. Of course these are not the only diagrams we must calculate; there is also the product of trees, which we will view as a cut box $B$, in the non-linear part of the colour kernel. Note that we now have two distinct colour structures to calculate, one for the loops and one for the cut box. We will investigate in detail how these structures affect the cancellation of classically singular terms, but first let us work with the 1-loop, linear piece.

\subsubsection{1-loop amplitude}

At NLO we need to calculate the 1-loop scalar amplitude

$$
\begin{aligned}
& \mathcal{A}^{(1)}=\mathcal{C}(\not) B+\mathcal{C}(\bigotimes) C+\mathcal{C}(\bigotimes) T_{12} \\
& +\mathcal{C}()_{21}+\mathcal{C}(\bigotimes) Y_{12}+\mathcal{C}\left(Y_{21}\right. \text {. }
\end{aligned}
$$

A first task is to choose a basis of independent colour structures. The complete set of colour factors can easily be calculated:

$$
\begin{aligned}
& \mathcal{C}(\mathcal{Z})=C_{1}^{a} C_{2}^{a} C_{1}^{b} C_{2}^{b}, \\
& \mathcal{C}(\text { Y })=C_{1}^{a} C_{2}^{b} C_{1}^{b} C_{2}^{a} \text {, } \\
& c(X)=\frac{1}{2} c(\not)+\frac{1}{2} c(\not)=c(\aleph) \text {, } \\
& \mathcal{C}(\rangle)=\hbar C_{1}^{a} f^{a b c} C_{2}^{b} C_{2}^{c}, \\
& \mathcal{C}(\triangleright)=\hbar C_{1}^{a} C_{1}^{b} f^{a b c} C_{2}^{c} \text {. }
\end{aligned}
$$

At first sight, we appear to have four independent colour factors: the box, cross box and the two non-Abelian triangles. However, it is very simple to see that the latter are in fact both proportional to the tree colour factor of equation (3.14); for example,

$$
\begin{aligned}
\mathcal{C}(\succ)=\frac{\hbar}{2} C_{1}^{a} f^{a b c}\left[C_{2}^{b}, C_{2}^{c}\right] & =\frac{i \hbar^{2}}{2} f^{a b c} f^{b c d} C_{1}^{a} C_{2}^{d} \\
& =\frac{i \hbar^{2}}{2} \mathcal{C}(\succ<),
\end{aligned}
$$

where we have used equation (2.10). Moreover, similar manipulations demonstrate that the cross-box colour factor is not in fact linearly independent:

$$
\begin{aligned}
\mathcal{C}(\mathbf{Y}) & =C_{1}^{a} C_{1}^{b}\left(C_{2}^{a} C_{2}^{b}-i \hbar f^{a b c} C_{2}^{c}\right) \\
& =\left(C_{1} \cdot C_{2}\right)\left(C_{1} \cdot C_{2}\right)-\frac{i \hbar}{2}\left[C_{1}^{a}, C_{2}^{b}\right] f^{a b c} C_{2}^{c} \\
& =\mathcal{C}(-C)+\frac{\hbar^{2}}{2} \mathcal{C}(\succ\langle) .
\end{aligned}
$$

Thus at 1-loop the classically significant part of the amplitude has a basis of two colour structures: the box and tree. Hence the decomposition of the 1-loop amplitude into partial 
amplitudes and colour structures is

$$
\begin{aligned}
\mathcal{A}^{(1)}= & \mathcal{C}(\neg)\left[B+C+T_{12}+T_{21}\right] \\
& +\frac{\hbar^{2}}{2} \mathcal{C}\left(\searrow<\left[C+\frac{T_{12}}{2}+\frac{T_{21}}{2}+i Y_{12}+i Y_{21}\right] .\right.
\end{aligned}
$$

This expression for the amplitude is particularly useful when taking the classical limit. The second term is proportional to two powers of $\hbar$ while the only possible singularity in $\hbar$ at one loop order is a factor $1 / \hbar$ in the evaluation of the kinematic parts of the diagrams. Thus, it is clear that the second line of the expression must be a quantum correction, and can be dropped in calculating the classical colour impulse. Perhaps surprisingly, these terms include the sole contribution from the non-Abelian triangles $Y_{i j}$, and thus we will not need to calculate these diagrams. We learn that classically, the 1-loop scalar YM amplitude has a basis of only one colour factor, and moreover depends on the same topologies as in electrodynamics, so we have

$$
\mathcal{A}^{(1)}=\mathcal{C}(\mathcal{Z}) \mathcal{A}^{(1, \mathrm{QED})}+\mathcal{O}(\hbar)
$$

in terms of the one-loop QED amplitude $\mathcal{A}^{(1, \mathrm{QED})}$.

The colour impulse factor in equation (3.19) therefore reduces to a single commutator. To calculate this we need to repeatedly apply the commutation relation in equation (2.10), which yields

$$
\begin{aligned}
{\left[C_{1}^{a}, \mathcal{C}(\text { Д })\right] } & =\left[C_{1}^{a}, C_{1}^{b} C_{1}^{c}\right] C_{2}^{b} C_{2}^{c} \\
& =i \hbar f^{a c d}\left(C_{1}^{d} C_{1}^{b} C_{2}^{c} C_{2}^{b}+C_{1}^{b} C_{1}^{d} C_{2}^{b} C_{2}^{c}\right) \\
& =i \hbar f^{a c d}\left(C_{1}^{d} C_{2}^{c}\left(C_{1} \cdot C_{2}\right)+\left(C_{1}^{d} C_{1}^{b}+i \hbar f^{b d e} C_{1}^{e}\right)\left(C_{2}^{c} C_{2}^{b}+i \hbar f^{b c e} C_{2}^{e}\right)\right) \\
& =i \hbar f^{a c d}\left(2 C_{1}^{d} C_{2}^{c}\left(C_{1} \cdot C_{2}\right)-i \hbar f^{d b e}\left(C_{1}^{e} C_{2}^{b} C_{2}^{c}-C_{2}^{e} C_{1}^{b} C_{1}^{c}\right)+\mathcal{O}\left(\hbar^{2}\right)\right) .
\end{aligned}
$$

The colour impulse factor is itself a series in $\hbar$. The partial amplitude is also a Laurent series in $\hbar$, which is presented in appendix B. In brief, the leading term in this expansion is the apparently singular (enhanced by one inverse power of $\hbar$ ) part $\mathcal{A}_{-1}^{(1, \mathrm{QED})} \sim \mathcal{O}\left(\hbar^{-2}\right)$, and the classical term $\mathcal{A}_{0}^{(1, \mathrm{QED})} \sim \mathcal{O}\left(\hbar^{-1}\right)$. This has a very important consequence for the impulse kernel - unlike in the QED case, the apparently singular term $\mathcal{A}_{-1}^{(1, \mathrm{QED})}$ in the partial amplitude now contributes classically, because of the second term in the colour impulse factor:

$$
\begin{aligned}
& \mathcal{G}_{1-\text { loop }}^{a,(1)}=\hbar g^{4}\left\{2 i f^{a c d} C_{1}^{d} C_{2}^{c}\left(C_{1} \cdot C_{2}\right)\left(\mathcal{A}_{-1}^{(1, \mathrm{QED})}+\mathcal{A}_{0}^{(1, \mathrm{QED})}\right)\right. \\
&\left.+\hbar f^{a c d} f^{d b e}\left(C_{1}^{e} C_{2}^{b} C_{2}^{c}-C_{2}^{e} C_{1}^{b} C_{1}^{c}\right) \mathcal{A}_{-1}^{(1, \mathrm{QED})}\right\} .
\end{aligned}
$$

However, there are still singular terms in the first line; their cancellation requires including the quadratic part of the colour kernel. 


\subsubsection{Cut box}

Rather than viewing the second term in equation (3.19) as a product of trees, we will treat the quadratic piece as a weighted cut of the box diagram, and define

$$
\begin{aligned}
B= & -i \hbar^{2} \int \hat{\mathrm{d}}^{4} \bar{\ell} \hat{\delta}\left(2 p_{1} \cdot \bar{\ell}+\hbar \bar{\ell}^{2}\right) \hat{\delta}\left(2 p_{2} \cdot \bar{\ell}-\hbar \bar{\ell}^{2}\right) \\
& \times \bar{A}_{\succ}\left(p_{1}+\hbar \bar{q}, p_{2}-\hbar \bar{q} \rightarrow p_{1}+\hbar \bar{\ell}, p_{2}-\hbar \bar{\ell}\right) \bar{A}_{\succ \prec}\left(p_{1}, p_{2} \rightarrow p_{1}+\hbar \bar{\ell}, p_{2}-\hbar \bar{\ell}\right) .
\end{aligned}
$$

Using the tree in equation (3.14) we can Laurent expand this expression in $\hbar$, as discussed in appendix B, which yields the leading terms

$$
\begin{aligned}
B_{-1} & =-i \frac{4\left(p_{1} \cdot p_{2}\right)^{2}}{\hbar^{2}} \int \hat{\mathrm{d}}^{4} \bar{\ell} \frac{\hat{\delta}\left(p_{1} \cdot \bar{\ell}\right) \hat{\delta}\left(p_{2} \cdot \bar{\ell}\right)}{\bar{\ell}^{2}(\bar{q}-\bar{\ell})^{2}} \\
B_{0} & =-i \frac{2\left(p_{1} \cdot p_{2}\right)^{2}}{\hbar} \int \hat{\mathrm{d}}^{4} \bar{\ell} \frac{\bar{\ell} \cdot \bar{q}}{\bar{\ell}^{2}(\bar{q}-\bar{\ell})^{2}}\left\{\hat{\delta}\left(p_{1} \cdot \bar{\ell}\right) \hat{\delta}^{\prime}\left(p_{2} \cdot \bar{\ell}\right)-\hat{\delta}\left(p_{2} \cdot \bar{\ell}\right) \hat{\delta}^{\prime}\left(p_{1} \cdot \bar{\ell}\right)\right\} .
\end{aligned}
$$

To determine the classical contributions we must calculate the associated colour impulse factor,

$$
\begin{aligned}
\mathcal{C}\left(>\langle)^{\dagger}\left[\mathbb{C}_{1}^{a}, \mathcal{C}(\rangle\langle)\right]\right. & =i \hbar\left(C_{1} \cdot C_{2}\right) f^{a b c} C_{1}^{c} C_{2}^{b} \\
& =i \hbar f^{a b c}\left(C_{1}^{c} C_{1}^{d}+i \hbar f^{d c e} C_{1}^{e}\right)\left(C_{2}^{b} C_{2}^{d}+i \hbar f^{d b e} C_{2}^{e}\right) \\
& =i \hbar f^{a c d} C_{1}^{d} C_{2}^{c}\left(C_{1} \cdot C_{2}\right)+\hbar^{2} f^{a c d} f^{d b e}\left(C_{1}^{e} C_{2}^{b} C_{2}^{c}-C_{2}^{e} C_{1}^{b} C_{1}^{c}\right)+\mathcal{O}\left(\hbar^{3}\right) .
\end{aligned}
$$

Clearly we have a similar situation to equation (3.28): the colour impulse factor is again an expansion in $\hbar$, and its leading term yields a classical contributions from $B_{0}$. Meanwhile $B_{-1}$ also contributes classically from the correction to the colour structure - however, there is still a singular term:

$$
\begin{aligned}
& \mathcal{G}_{\text {cut box }}^{a,(1)}=i \hbar g^{4} f^{a c d}( C_{1}^{d} C_{2}^{c}\left(C_{1} \cdot C_{2}\right)\left(B_{-1}+B_{0}\right) \\
&-\left.i \hbar f^{d b e}\left(C_{1}^{e} C_{2}^{b} C_{2}^{c}-C_{2}^{e} C_{1}^{b} C_{1}^{c}\right) B_{-1}\right) \\
&=2 g^{4} f^{a c d}\left(p_{1} \cdot p_{2}\right)^{2} \int \hat{\mathrm{d}}^{4} \bar{\ell} \frac{1}{\bar{\ell}^{2}(\bar{q}-\bar{\ell})^{2}}\left\{C_{1}^{d} C_{2}^{c}\left(C_{1} \cdot C_{2}\right)\right. \\
& \times\left[\frac{2 \hat{\delta}\left(p_{1} \cdot \bar{\ell}\right) \hat{\delta}\left(p_{2} \cdot \bar{\ell}\right)}{\hbar}+\bar{\ell} \cdot \bar{q}\left(\hat{\delta}\left(p_{1} \cdot \bar{\ell}\right) \hat{\delta}^{\prime}\left(p_{2} \cdot \bar{\ell}\right)-\hat{\delta}\left(p_{2} \cdot \bar{\ell}\right) \hat{\delta}^{\prime}\left(p_{1} \cdot \bar{\ell}\right)\right)\right] \\
&\left.-2 i f^{d b e}\left(C_{1}^{e} C_{2}^{b} C_{2}^{c}-C_{2}^{e} C_{1}^{b} C_{1}^{c}\right) \hat{\delta}\left(p_{1} \cdot \bar{\ell}\right) \hat{\delta}\left(p_{2} \cdot \bar{\ell}\right)\right\} .
\end{aligned}
$$

\subsubsection{Combining terms}

It now remains to calculate the full colour kernel,

$$
\mathcal{G}^{a,(1)}=\mathcal{G}_{1-\text { loop }}^{a,(1)}+\mathcal{G}_{\text {cut box }}^{a,(1)} .
$$

The first priority is to study the classically singular terms which sit in both parts of the kernel. Recall that each part of equation (3.19) came with a different colour kernel. The 
upshot of this fact is that, after explicit calculation, the singular terms now involve one common colour structure:

$$
\begin{aligned}
\mathcal{G}_{-1}^{a,(1)}= & i \hbar g^{4} f^{a c d} C_{1}^{d} C_{2}^{c}\left(C_{1} \cdot C_{2}\right)\left(2 \mathcal{A}_{-1}^{(1, \mathrm{QED})}+B_{-1}\right) \\
& +\hbar^{2} g^{4} f^{a c d} f^{d b e}\left(C_{1}^{e} C_{2}^{b} C_{2}^{c}-C_{2}^{e} C_{1}^{b} C_{1}^{c}\right)\left(\mathcal{A}_{-1}^{(1, \mathrm{QED})}+B_{-1}\right) .
\end{aligned}
$$

As detailed in appendix B, the singular term in the expansion of the QED amplitude originates entirely from box diagrams, and takes a neat form in terms of delta functions:

$$
\mathcal{A}_{-1}^{(1, \mathrm{QED})}=i \frac{2\left(p_{1} \cdot p_{2}\right)^{2}}{\hbar^{2}} \int \hat{\mathrm{d}}^{4} \bar{\ell} \frac{1}{\bar{\ell}^{2}(\bar{q}-\bar{\ell})^{2}} \hat{\delta}\left(p_{1} \cdot \bar{\ell}\right) \hat{\delta}\left(p_{2} \cdot \bar{\ell}\right) .
$$

This expression is the same as the $B_{-1}$ term in equation (3.30). The coefficients are such that the terms in the first line of equation (3.34) cancel, ensuring the apparently singular part of the colour kernel vanishes.

However, an interesting new feature of the colour impulse is that the colour structure in the second line of equation (3.34) combines with the sum of the 1-loop singular terms to give a non-zero classical contribution:

$$
\left[\mathcal{G}_{-1}^{a,(1)}\right]_{\mathcal{O}\left(\hbar^{0}\right)}=-2 i g^{4} f^{a c d} f^{d b e}\left(C_{1}^{e} C_{2}^{b} C_{2}^{c}-C_{2}^{e} C_{1}^{b} C_{2}^{c}\right)\left(p_{1} \cdot p_{2}\right)^{2} \int \hat{\mathrm{d}}^{4} \bar{\ell} \frac{\hat{\delta}\left(p_{1} \cdot \bar{\ell}\right) \hat{\delta}\left(p_{2} \cdot \bar{\ell}\right)}{\bar{\ell}^{2}(\bar{q}-\bar{\ell})^{2}}
$$

With all possible singular terms safely dealt with, it remains to combine the $\mathcal{O}\left(\hbar^{0}\right)$ terms in equation (3.28) and equation (3.32). Conveniently, these all have the same colour factor:

$$
\mathcal{G}^{a,(1)}=i g^{4} \hbar f^{a c d} C_{1}^{d} C_{2}^{c}\left(C_{1} \cdot C_{2}\right)\left(2 \mathcal{A}_{0}^{(1, \mathrm{QED})}+B_{0}\right)+\left[\mathcal{G}_{-1}^{a,(1)}\right]_{\mathcal{O}\left(\hbar^{0}\right)} .
$$

Now we can sum the diagrams in the partial amplitude, the explicit expressions for which are given in appendix B. The result is

$$
\begin{aligned}
\mathcal{G}^{a,(1)}=g^{4} \int \hat{\mathrm{d}}^{4} \bar{\ell} \frac{1}{\bar{\ell}^{2}(\bar{\ell}-\bar{q})^{2}} & \left\{4 i f^{a c d} C_{2}^{c} C_{1}^{d}\left(C_{1} \cdot C_{2}\right)\right. \\
\times & {\left[\hat{\delta}\left(p_{1} \cdot \bar{\ell}\right)\left[m_{1}^{2}+\left(p_{1} \cdot p_{2}\right)^{2} \bar{\ell} \cdot(\bar{\ell}-\bar{q})\left(\frac{1}{\left(p_{2} \cdot \bar{\ell}-i \epsilon\right)^{2}}+i \hat{\delta}^{\prime}\left(p_{2} \cdot \bar{\ell}\right)\right)\right]\right.} \\
+ & \left.\hat{\delta}\left(p_{2} \cdot \bar{\ell}\right)\left[m_{2}^{2}+\left(p_{1} \cdot p_{2}\right)^{2} \bar{\ell} \cdot(\bar{\ell}-\bar{q})\left(\frac{1}{\left(p_{1} \cdot \bar{\ell}+i \epsilon\right)^{2}}-i \hat{\delta}^{\prime}\left(p_{1} \cdot \bar{\ell}\right)\right)\right]\right] \\
- & \left.2 i f^{a c d} f^{d b e}\left(C_{1}^{e} C_{2}^{b} C_{2}^{c}-C_{2}^{e} C_{1}^{b} C_{1}^{c}\right)\left(p_{1} \cdot p_{2}\right)^{2} \hat{\delta}\left(p_{1} \cdot \bar{\ell}\right) \hat{\delta}\left(p_{2} \cdot \bar{\ell}\right)\right\} .
\end{aligned}
$$

\subsubsection{Final result}

Finally the observable, the colour impulse, is given by

$$
\Delta c_{1}^{a,(1)}=\left\langle\left\langle i \int \hat{\mathrm{d}}^{4} \bar{q} \hat{\delta}\left(2 p_{1} \cdot \bar{q}\right) \hat{\delta}\left(2 p_{2} \cdot \bar{q}\right) e^{-i b \cdot \bar{q}} \mathcal{G}^{a,(1)}\right\rangle .\right.
$$


Upon substituting the kernel in equation (3.38), we can average over the momentum and colour wavefunctions implicit in the expectation value. For sharply peaked momentum wavefunctions and large $\mathrm{SU}(N)$ representations, this merely has the effect of sending $p_{i} \mapsto$ $m_{i} u_{i}$, and replacing quantum colour factors with products of commuting classical charges. Hence we finally obtain the NLO colour impulse

$$
\begin{aligned}
\Delta c_{1}^{a,(1)}= & g^{4} \int \hat{\mathrm{d}}^{4} \bar{q} \hat{\mathrm{d}}^{4} \bar{\ell} \hat{\delta}\left(u_{1} \cdot \bar{q}\right) \hat{\delta}\left(u_{2} \cdot \bar{q}\right) e^{-i \bar{q} \cdot b} \frac{1}{\overline{\ell^{2}(\bar{\ell}-\bar{q})^{2}}} \\
& \times\left\{\hat { \delta } ( u _ { 1 } \cdot \overline { \ell } ) \left[\frac { f ^ { a c d } c _ { 1 } ^ { c } c _ { 2 } ^ { d } ( c _ { 1 } \cdot c _ { 2 } ) } { m _ { 2 } } \left[1+\left(u_{1} \cdot u_{2}\right)^{2} \bar{\ell} \cdot(\bar{\ell}-\bar{q})\left(\frac{1}{\left(u_{2} \cdot \bar{\ell}-i \epsilon\right)^{2}}\right.\right.\right.\right. \\
& \left.\left.\left.\quad+i \hat{\delta}^{\prime}\left(u_{2} \cdot \bar{\ell}\right)\right)\right]-f^{a c d} f^{d b e} c_{1}^{b} c_{1}^{c} c_{2}^{e} \frac{\left(u_{1} \cdot u_{2}\right)^{2}}{2} \hat{\delta}\left(u_{2} \cdot \bar{\ell}\right)\right] \\
& +\hat{\delta}\left(u_{2} \cdot \bar{\ell}\right)\left[\frac { f ^ { a c d } c _ { 1 } ^ { c } c _ { 2 } ^ { d } ( c _ { 1 } \cdot c _ { 2 } ) } { m _ { 1 } } \left[1+\left(u_{1} \cdot u_{2}\right)^{2} \bar{\ell} \cdot(\bar{\ell}-\bar{q})\left(\frac{1}{\left(u_{1} \cdot \bar{\ell}+i \epsilon\right)^{2}}\right.\right.\right. \\
& \left.\left.\left.\left.\quad-i \hat{\delta}^{\prime}\left(u_{1} \cdot \bar{\ell}\right)\right)\right]+f^{a c d} f^{d b e} c_{1}^{e} c_{2}^{b} c_{2}^{c} \frac{\left(u_{1} \cdot u_{2}\right)^{2}}{2} \hat{\delta}\left(u_{1} \cdot \bar{\ell}\right)\right]\right\} .
\end{aligned}
$$

This is found to agree with the result obtained by solving the classical equations of motion, which is discussed in section 5 .

\subsection{Momentum}

It is evident that the usual (momentum) impulse in YM theory should be similar to the QED case discussed in [15]. But it is also natural to expect some new terms in the YM impulse in view of the self-coupling of the YM field. Diagrams involving this self-coupling are present at NLO. In this subsection, we investigate the impulse in the YM case with this thought in mind. We begin with equation (3.12) for the impulse kernel $\mathcal{I}^{\mu}$, which now only involves colour factors of the partial amplitudes themselves.

At leading order we can just reuse the expressions in equation (3.14), finding

$$
\mathcal{I}^{\mu,(0)}=4 g^{2} \frac{\left(p_{1} \cdot p_{2}\right)}{\bar{q}^{2}} \bar{q}^{\mu} C_{1} \cdot C_{2}
$$

Then, substituting into equation (3.11) and taking the classical limit as before we have the LO momentum impulse

$$
\Delta p_{1}^{\mu,(0)}=i g^{2} c_{1} \cdot c_{2} \int \hat{\mathrm{d}}^{4} \bar{q} \frac{\hat{\delta}\left(u_{1} \cdot \bar{q}\right) \hat{\delta}\left(u_{2} \cdot \bar{q}\right) e^{-i b \cdot \bar{q}}}{\bar{q}^{2}}\left(u_{1} \cdot u_{2}\right) \bar{q}^{\mu} .
$$

This expression is closely related to the NLO impulse in QED, which can be obtained from the YM case by replacing $c_{1} \cdot c_{2}$ with the product of the electric charges of the two particles. This relationship is natural, since at leading order the gluons do not self-interact. 
Just as in the colour case, at NLO the momentum kernel has linear, 1-loop and quadratic, cut box components:

$$
\begin{aligned}
\mathcal{I}^{\mu,(1)}= & \hbar g^{4} \bar{q}^{\mu} \sum_{\Gamma} \mathcal{C}(\Gamma) \bar{A}_{\Gamma}^{(1)}\left(p_{1}, p_{2} \rightarrow p_{1}+\hbar \bar{q}, p_{2}-\hbar \bar{q}\right) \\
& -i g^{4} \hbar^{3} \int \hat{\mathrm{d}}^{4} \bar{\ell} \hat{\delta}\left(2 p_{1} \cdot \bar{\ell}+\hbar \bar{\ell}^{2}\right) \hat{\delta}\left(2 p_{2} \cdot \bar{\ell}-\hbar \bar{\ell}^{2}\right) \bar{\ell}^{\mu} \mathcal{C}(\succ \measuredangle)^{\dagger} \mathcal{C}(\succ \prec) \\
& \times \bar{A}_{\succ}\left(p_{1}+\hbar \bar{q}, p_{2}-\hbar \bar{q} \rightarrow p_{1}+\hbar \bar{\ell}, p_{2}-\hbar \bar{\ell}\right) \bar{A}_{\succ}\left(p_{1}, p_{2} \rightarrow p_{1}+\hbar \bar{\ell}, p_{2}-\hbar \bar{\ell}\right) .
\end{aligned}
$$

The decomposition of the 1-loop amplitude onto the colour basis in equation (3.25) makes computing the first term (linear in the one-loop amplitude) in the impulse kernel trivial; we have

$$
\mathcal{I}_{1-\text { loop }}^{\mu,(1)}=\hbar g^{4} \bar{q}^{\mu} \mathcal{C}(\mathcal{Z})\left(\mathcal{A}_{-1}^{(1, \mathrm{QED})}+\mathcal{A}_{0}^{(1, \mathrm{QED})}\right) .
$$

This means that the non-Abelian triangle Feynman diagrams do not contribute to the impulse: a somewhat surprising result, since it is only in these diagrams that the selfinteraction of the gluons appears.

Meanwhile we will denote the kinematic terms in the quadratic piece of the momentum kernel $B^{\mu}$, which has the same definition as equation (3.29) but dressed with an extra loop momentum - explicit expressions are given in appendix B. Its colour factor is simply

$$
\mathcal{C}(\rangle\langle)^{\dagger} \mathcal{C}(\rangle\langle)=\left(C_{2} \cdot C_{1}\right)\left(C_{1} \cdot C_{2}\right)=\mathcal{C}(\not)
$$

Thus there is only one relevant colour structure in the NLO momentum impulse, that of the box. The momentum kernel factorises accordingly:

$$
\mathcal{I}^{\mu,(1)}=g^{4}\left(C_{1} \cdot C_{2}\right)^{2}\left[\hbar \bar{q}^{\mu}\left(\mathcal{A}_{-1}^{(1, \mathrm{QED})}+\mathcal{A}_{0}^{(1, \mathrm{QED})}\right)+B_{-1}^{\mu}+B_{0}^{\mu}\right] .
$$

This is just a colour dressing of the NLO momentum impulse in QED — in particular, the cancellation of singular terms between the cut box and 1-loop diagrams is guaranteed [15]. Gathering all the terms from triangles, boxes and the cut box in appendix B and inserting into equation (3.11), upon taking the classical limit in the now familiar way we find

$$
\begin{aligned}
& \Delta p_{1}^{\mu,(1)}=\frac{g^{4}\left(c_{1} \cdot c_{2}\right)^{2}}{2} \int \hat{\mathrm{d}}^{4} \bar{\ell}^{4} \bar{q} \frac{\hat{\delta}\left(u_{1} \cdot \bar{q}\right) \hat{\delta}\left(u_{2} \cdot \bar{q}\right)}{\bar{\ell}^{2}(\bar{\ell}-\bar{q})^{2}}\left[\overline { q } ^ { \mu } \left\{\frac{\hat{\delta}\left(u_{2} \cdot \bar{\ell}\right)}{m_{1}}+\frac{\hat{\delta}\left(u_{1} \cdot \bar{\ell}\right)}{m_{2}}\right.\right. \\
& \left.+\left(u_{1} \cdot u_{2}\right)^{2} \bar{\ell} \cdot(\bar{\ell}-\bar{q})\left(\frac{\hat{\delta}\left(u_{1} \cdot \bar{\ell}\right)}{m_{2}\left(u_{2} \cdot \bar{\ell}-i \epsilon\right)^{2}}+\frac{\hat{\delta}\left(u_{2} \cdot \bar{\ell}\right)}{m_{1}\left(u_{1} \cdot \bar{\ell}+i \epsilon\right)^{2}}\right)\right\} \\
& \left.-i \overline{\ell^{\mu}} \bar{\ell} \cdot(\bar{\ell}-\bar{q})\left(\frac{\hat{\delta}^{\prime}\left(u_{1} \cdot \bar{\ell}\right) \hat{\delta}\left(u_{2} \cdot \bar{\ell}\right)}{m_{2}}-\frac{\hat{\delta}\left(u_{1} \cdot \bar{\ell}\right) \hat{\delta}^{\prime}\left(u_{2} \cdot \bar{\ell}\right)}{m_{1}}\right)\right] \text {. }
\end{aligned}
$$

We have found that in the non-Abelian theory the final result for the impulse is identical to QED [15] with the charge to colour replacement $Q_{1} Q_{2} \rightarrow c_{1} \cdot c_{2}$. In fact this result follows from the colour basis decomposition in equation (3.25) and in particular the fact that the non-Abelian triangle diagrams only contribute to the $\hbar^{2}$ suppressed second colour structure. 


\section{Radiation}

One of the strengths of studying impulse-like observables is that radiative phenomena are naturally included, as explored in depth in [15]. Moreover, the double copy makes radiation in Yang-Mills theory a powerful tool for studying its gravitational counterpart $[11,21,85]$. The general discussion of radiation of momentum in KMOC [15] applies directly to the Yang-Mills case, but weakly-coupled YM theory contains another interesting observable: the total colour radiated to infinity. In this section, we study this radiation of colour in the quantum formalism. As an explicit example we compute the leading order classical colour current found in [11] from scattering amplitudes.

\subsection{Total radiated colour}

The construction and calculation of the colour impulse relied on the adjoint-valued colour operator in equation (2.9) for a scalar field in representation $R$. To study the total radiated colour we need a similar operator for the gluon radiation field. This can easily be obtained by restricting to the adjoint representation, namely by taking $\left(T_{\text {adj }}^{a}\right)_{b}{ }^{c}=i f^{b a c}$. Since the gluon field is real and has two helicity eigenstates, its colour operator is

$$
\mathbb{F}^{a}=i \hbar f^{b a c} \sum_{\sigma= \pm} \int \mathrm{d} \Phi(k) a_{\sigma}^{b \dagger}(k) a_{\sigma}^{c}(k)
$$

where $\sigma$ labels the helicity. Of course, it is also possible to derive this expression directly from the Noether charge for vector fields in the adjoint representation, given in equation (5.15), in close analogy to our discussion in sections 2.1 and 2.2.

This adjoint colour charge is of interest elsewhere in the literature since it plays a role in the physics of YM theory at asymptotic infinity [106-112]. In this connection, the nature of the final state of the radiation is relevant [111]. Here, we do not compute this final state explicitly. Instead, we compute expectation values of operators on the final state.

Following the KMOC route [15] to obtain an expression for the total colour charge radiated from a scattering event leads to

$$
\left\langle R_{\mathrm{col}}^{a}\right\rangle=\left\langle\Psi\left|T^{\dagger} \mathbb{F}^{a} T\right| \Psi\right\rangle,
$$

where we made use of the fact that there are no gauge bosons in the incoming state of equation (2.35). Before expanding in terms of on-shell scattering amplitudes, it is worth demonstrating colour conservation in our formalism. At the operator level, assuming only the quantum fields corresponding to particles 1 and 2 are present in addition to the YangMills field, the statement that colour is conserved is

$$
\left[\mathbb{C}_{1}^{a}+\mathbb{C}_{2}^{a}+\mathbb{F}^{a}, T\right]=0
$$

It then immediately follows that

$$
\begin{aligned}
\left\langle\Delta c_{1}^{a}\right\rangle+\left\langle\Delta c_{2}^{a}\right\rangle & =\left\langle\Psi\left|T^{\dagger}\left[\mathbb{C}_{1}^{a}, T\right]\right| \Psi\right\rangle+\left\langle\Psi\left|T^{\dagger}\left[\mathbb{C}_{2}^{a}, T\right]\right| \Psi\right\rangle \\
& =-\left\langle\Psi\left|T^{\dagger}\left[\mathbb{F}^{a}, T\right]\right| \Psi\right\rangle=-\left\langle\Psi\left|T^{\dagger} \mathbb{F}^{a} T\right| \Psi\right\rangle=-\left\langle R_{\mathrm{col}}^{a}\right\rangle
\end{aligned}
$$


where the second line holds from the absence of gluon radiation in the incoming state. Total colour is therefore conserved in the quantum theory, as it must be given the associated global symmetry.

Let us proceed in expanding equation (4.2) by inserting complete sets of states; for leading order radiation we need to consider an extra explicit gluon with momentum $k$ and a colour index, so we will take the set $X$ in the resolution of identity in equation (3.3) to just include the contribution

$$
\sum_{b, \sigma} \int \mathrm{d} \Phi(k)\left|k^{b}, \sigma\right\rangle\left\langle k^{b}, \sigma\right|
$$

Note that higher order corrections could also be obtained by adding further states, but we will just be interested in the lowest order case here. Using equation (4.1) and integrating over intermediate delta functions we find

$$
\begin{aligned}
\left\langle R_{\mathrm{col}}^{a}\right\rangle= & \sum_{b, c, \sigma} \int \mathrm{d} \Phi(k) \mathrm{d} \Phi(\tilde{k}) \mathrm{d} \Phi\left(r_{1}\right) \mathrm{d} \Phi\left(r_{2}\right) \mathrm{d} \mu\left(\zeta_{1}\right) \mathrm{d} \mu\left(\zeta_{2}\right) \\
& \times\left\langle\Psi\left|T^{\dagger}\right| r_{1} r_{2} k^{b}, \sigma ; \zeta_{1} \zeta_{2}\right\rangle\left\langle k^{b}, \sigma\left|\mathbb{F}^{a}\right| \tilde{k}^{c}, \sigma\right\rangle\left\langle r_{1} r_{2} \tilde{k}^{c}, \sigma ; \zeta_{1} \zeta_{2}|T| \Psi\right\rangle \\
= & -i \hbar \sum_{b, c, \sigma} \int \mathrm{d} \Phi(k) \mathrm{d} \Phi\left(r_{1}\right) \mathrm{d} \Phi\left(r_{2}\right) \mathrm{d} \mu\left(\zeta_{1}\right) \mathrm{d} \mu\left(\zeta_{2}\right) f^{a b c} \Upsilon^{* b}\left(r_{1}, r_{2} ; k, \sigma\right) \Upsilon^{c}\left(r_{1}, r_{2} ; k, \sigma\right),
\end{aligned}
$$

where

$$
\begin{aligned}
\Upsilon^{a}\left(r_{1}, r_{2} ; k, \sigma\right)= & \int \mathrm{d} \Phi\left(p_{1}\right) \mathrm{d} \Phi\left(p_{2}\right) \phi_{1}\left(p_{1}\right) \phi_{2}\left(p_{2}\right) e^{i b \cdot p_{1} / \hbar} \hat{\delta}^{(4)}\left(p_{1}+p_{2}-r_{1}-r_{2}-k\right) \\
& \times \sum_{D}\left\langle\zeta_{1} \zeta_{2}\left|\mathcal{C}^{a}(D)\right| \chi_{1} \chi_{2}\right\rangle A_{D}\left(p_{1}, p_{2} \rightarrow r_{1}, r_{2} ; k, \sigma\right) .
\end{aligned}
$$

We have factorised the amplitude into colour structures $\mathcal{C}(D)$ and partial amplitudes $A_{D}$, as in equation (3.4). However, here the colour factor gains a free index from the external gluon state.

To take the classical limit of equation (4.6) and introduce radiation kernels we follow [15], finding

$$
R_{\text {col }}^{a}=-i f^{a b c} \sum_{\sigma}\left\langle\left\langle\hbar^{-2} \int \mathrm{d} \Phi(k) \mathcal{R}^{* b}(k, \sigma) \mathcal{R}^{c}(k, \sigma)\right\rangle\right\rangle .
$$

The large angle brackets, defined in equation (3.8), are the expectation value over the incoming scalar wavepackets, and thus include the colour states. The radiation kernels inherit the colour index of the external gluon, and take the form

$$
\begin{aligned}
\mathcal{R}^{a}(k, \sigma)= & \hbar^{\frac{3}{2}} \int \hat{\mathrm{d}}^{4} q_{1} \hat{\mathrm{d}}^{4} q_{2} \hat{\delta}\left(2 p_{1} \cdot q_{1}+q_{1}^{2}\right) \hat{\delta}\left(2 p_{2} \cdot q_{2}+q_{2}^{2}\right) \hat{\delta}^{(4)}\left(k-q_{1}-q_{2}\right) e^{i b \cdot q_{1} / \hbar} \\
& \times \sum_{D} \mathcal{C}^{a}(D) A_{D}\left(p_{1}+q_{1}, p_{2}+q_{2} \rightarrow p_{1}, p_{2} ; k, \sigma\right) .
\end{aligned}
$$

The powers of $\hbar$ are organised such that the radiation kernel will be $\mathcal{O}\left(\hbar^{0}\right)$ and therefore classical in the limit. Note that because the colour charge has dimensions of angular momentum, the $\hbar$ scaling here works out the same way as in the total radiated momentum. 


\subsection{Leading order evaluation}

Let us explicitly compute the leading order radiation kernel for the scattering of two massive scalar particles, described by the action in equation (2.1). In the classical limit the LO kernel is given in terms of coupling constant stripped amplitudes by

$$
\begin{aligned}
\mathcal{R}^{a,(0)}(\bar{k})= & \hbar^{2} g^{3} \int \hat{\mathrm{d}}^{4} \bar{q}_{1} \hat{\mathrm{d}}^{4} \bar{q}_{2} \hat{\delta}\left(2 p_{1} \cdot \bar{q}_{1}+\hbar \bar{q}_{1}^{2}\right) \hat{\delta}\left(2 p_{2} \cdot \bar{q}_{2}+\hbar \bar{q}_{2}^{2}\right) \\
& \times \hat{\delta}^{(4)}\left(\bar{k}-\bar{q}_{1}-\bar{q}_{2}\right) e^{i b \cdot \bar{q}_{1}} \sum_{D} \mathcal{C}^{a}(D) \bar{A}_{D}^{(0)}\left(p_{1}+q_{1}, p_{2}+q_{2} \rightarrow p_{1}, p_{2} ; k, \sigma\right) .
\end{aligned}
$$

Clearly the terms in the amplitude which contribute to the classical radiation are those at $\mathcal{O}\left(\hbar^{-2}\right)$. The shifts in the delta functions are important for obtaining this accurately. The relevant amplitude is the non-Abelian extension of the 5-point tree studied in [15]; this was used in [21] to take the double copy and calculate radiation in Einstein gravity. The relevant Feynman topologies for emission from particle 1 are $^{8}$
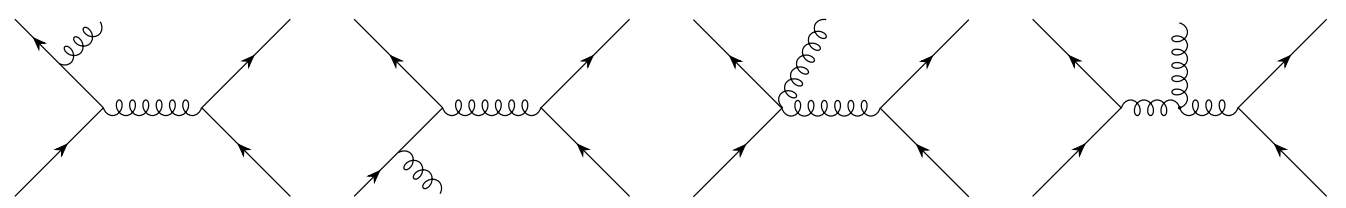

so we need to calculate the classical terms in the 5-point amplitude

$$
\begin{aligned}
& \mathcal{A}^{(0)}\left(\bar{k}^{a}\right)=\sum_{D} \mathcal{C}^{a}(D) \bar{A}_{D}^{(0)}\left(p_{1}+q_{1}, p_{2}+q_{2} \rightarrow p_{1}, p_{2} ; k, \sigma\right)
\end{aligned}
$$

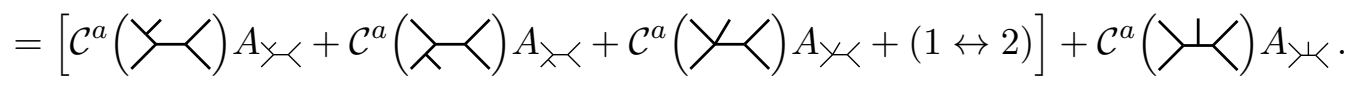

Explicitly, the colour factors are given by

$$
\begin{aligned}
& \mathcal{C}^{a}(Y \prec)=\left(C_{1}^{a} \cdot C_{1}^{b}\right) C_{2}^{b}, \quad \quad \mathcal{C}^{a}\left(\succ\left(C_{1}^{b} \cdot C_{1}^{a}\right) C_{2}^{b},\right. \\
& \mathcal{C}^{a}(Y<)=\frac{1}{2} \mathcal{C}^{a}(Y \prec)+\frac{1}{2} \mathcal{C}^{a}(\succ \prec), \quad \mathcal{C}^{a}(\bigvee)=\hbar f^{a b c} C_{1}^{b} C_{2}^{c},
\end{aligned}
$$

with the replacement $1 \leftrightarrow 2$ for diagrams with gluon emission from particle 2 . Just as in the 4-point case at 1-loop, this is an overcomplete set for specifying a basis, because

$$
\mathcal{C}^{a}(\lambda \prec)=\left(C_{1}^{a} \cdot C_{1}^{b}\right) C_{2}^{b}+i \hbar f^{b a c} C_{1}^{c} C_{2}^{b}=\mathcal{C}^{a}(Y \prec)+i \mathcal{C}^{a}(\curlywedge)
$$

Hence the full basis of colour factors is only 3 dimensional, and the colour decomposition of the 5-point tree is

$$
\begin{aligned}
& \mathcal{A}^{(0)}\left(\bar{k}^{a}\right)=\mathcal{C}^{a}(Y \prec)\left(A_{\curlyvee \prec}+A_{\succ \prec}+A_{\searrow<}\right) \\
& +\frac{1}{2} \mathcal{C}^{a}(\searrow \prec)\left(A_{\succ \prec}+2 i A_{\succ}+i A_{\curlyvee}\right)+(1 \leftrightarrow 2) .
\end{aligned}
$$

\footnotetext{
${ }^{8}$ The momentum routing is as indicated in equation (4.10).
} 
Given that the second structure is $\mathcal{O}(\hbar)$, it would appear that we could again neglect the second term as a quantum correction. However, this intuition is not quite correct, as calculating the associated partial amplitude shows:

$$
\begin{aligned}
A_{\succ}+2 i A_{\succ}+i A_{\curlyvee}=-\frac{4 i \varepsilon_{\mu}^{h}(\bar{k})}{\hbar^{2}}\left[\frac{2 p_{1} \cdot p_{2}}{\bar{q}_{2}^{2} p_{1} \cdot \bar{k}} \frac{p_{1}^{\mu}}{\hbar}+\frac{1}{\hbar \bar{q}_{1}^{2} \bar{q}_{2}^{2}}\left(2 p_{2} \cdot \bar{k} p_{1}^{\mu}\right.\right. \\
\left.\left.-p_{1} \cdot p_{2}\left(\bar{q}_{1}^{\mu}-\bar{q}_{2}^{\mu}\right)-2 p_{1} \cdot \bar{k} p_{2}^{\mu}\right)+\mathcal{O}\left(\hbar^{0}\right)\right],
\end{aligned}
$$

where we have used $p_{1} \cdot \bar{q}_{2}=p_{1} \cdot \bar{k}-\hbar \bar{q}_{1}^{2} / 2$ on the support of the on-shell delta functions in the kernel equation (4.10). The partial amplitude appears to be singular, as there is an extra power of $\hbar$ downstairs. However, this will cancel against the extra power in the colour structure, yielding a classical contribution. Meanwhile in the other partial amplitude the potentially singular terms cancel trivially, as in QED, and the contribution is classical:

$$
\begin{aligned}
A_{\curlyvee<+}+A_{\succ \prec}+A_{\curlyvee<}=\frac{2}{\hbar^{2}} \frac{\varepsilon_{\mu}^{h}(\bar{k})}{\bar{q}_{2}^{2} p_{1} \cdot \bar{k}} & {\left[2 p_{1} \cdot p_{2} \bar{q}_{2}^{\mu}+\frac{p_{1} \cdot p_{2}}{p_{1} \cdot \bar{k}} p_{1}^{\mu}\left(\bar{q}_{1}^{2}-\bar{q}_{2}^{2}\right)\right.} \\
& \left.-2 p_{1} \cdot \bar{k} p_{2}^{\mu}+2 p_{2} \cdot \bar{k} p_{1}^{\mu}+\mathcal{O}(\hbar)\right] .
\end{aligned}
$$

Summing all colour factors and partial amplitudes, the classically significant part of the 5 -point amplitude is

$$
\begin{aligned}
& \overline{\mathcal{A}}^{(0)}\left(\bar{k}^{a}\right)= \sum_{D} \mathcal{C}^{a}(D) \\
&=-\frac{4 \varepsilon_{\mu}^{h}(\bar{k})}{\hbar^{2}}\left\{\frac{{ }^{(0)}(\bar{k})}{\bar{q}_{2}^{a}\left(C_{1} \cdot C_{2}\right)}\left[-\left(p_{1} \cdot p_{2}\right)\left(\bar{q}_{2}^{\mu}-\frac{\bar{k} \cdot \bar{q}_{2}}{\bar{k} \cdot p_{1}} p_{1}^{\mu}\right)+\bar{k} \cdot p_{1} p_{2}^{\mu}-\bar{k} \cdot p_{2} p_{1}^{\mu}\right]\right. \\
&\left.\quad+\frac{i f^{a b c} C_{1}^{b} C_{2}^{c}}{\bar{q}_{1}^{2} \bar{q}_{2}^{2}}\left[2 \bar{k} \cdot p_{2} p_{1}^{\mu}-p_{1} \cdot p_{2} \bar{q}_{1}^{\mu}+p_{1} \cdot p_{2} \frac{\bar{q}_{1}^{2}}{\bar{k} \cdot p_{1}} p_{1}^{\mu}\right]+(1 \leftrightarrow 2)\right\},
\end{aligned}
$$

where we have used that $\bar{q}_{1}^{2}-\bar{q}_{2}^{2}=-2 \bar{k} \cdot \bar{q}_{2}$ since the outgoing radiation is on-shell. Finally, we can substitute into the radiation kernel in equation (4.10) and take the classical limit. Averaging over the wavepackets sets $p_{i}=m_{i} u_{i}$ and replaces quantum colour charges with their classical counterparts, yielding

$$
\begin{aligned}
\mathcal{R}^{a,(0)}(\bar{k})= & -g^{3} \int \hat{\mathrm{d}}^{4} \bar{q}_{1} \hat{\mathrm{d}}^{4} \bar{q}_{2} \hat{\delta}^{(4)}\left(\bar{k}-\bar{q}_{1}-\bar{q}_{2}\right) \hat{\delta}\left(u_{1} \cdot \bar{q}_{1}\right) \hat{\delta}\left(u_{2} \cdot \bar{q}_{2}\right) e^{i b \cdot \bar{q}_{1}} \varepsilon_{\mu}^{h} \\
& \times\left\{\frac{c_{1} \cdot c_{2}}{m_{1}} \frac{c_{1}^{a}}{\bar{q}_{2}^{2} \bar{k} \cdot u_{1}}\left[-\left(u_{1} \cdot u_{2}\right)\left(\bar{q}_{2}^{\mu}-\frac{\bar{k} \cdot \bar{q}_{2}}{\bar{k} \cdot u_{1}} u_{1}^{\mu}\right)+\bar{k} \cdot u_{1} u_{2}^{\mu}-\bar{k} \cdot u_{2} u_{1}^{\mu}\right]\right. \\
& \left.+\frac{i f^{a b c} c_{1}^{b} c_{2}^{c}}{\bar{q}_{1}^{2} \bar{q}_{2}^{2}}\left[2 \bar{k} \cdot u_{2} u_{1}^{\mu}-u_{1} \cdot u_{2} \bar{q}_{1}^{\mu}+u_{1} \cdot u_{2} \frac{\bar{q}_{1}^{2}}{\bar{k} \cdot u_{1}} u_{1}^{\mu}\right]+(1 \leftrightarrow 2)\right\} .
\end{aligned}
$$

Our result is equal to the leading order current $\tilde{K}^{a,(0)}$ obtained in [11] by iteratively solving the Wong equations in equation (1.1a) and equation (1.1b) for timelike particle worldlines. We will show this explicitly in the next section. 


\section{Classical perspectives}

In this section we compute the same classical observables, impulse and radiation, using purely classical techniques. These calculations are not too complex, and serve to verify the results we obtained using scattering amplitudes. This gives confidence in applying these quantum methods to gravity, for example, where the classical calculations can become significantly more involved. We start with the colour and momentum impulses before moving to the total radiated colour charge, discussing its relation to asymptotic symmetries.

\subsection{Impulses from equations of motion}

We start with the NLO colour impulse, initially in the more general case of a system of $N$ interacting particles, and later restrict to the $N=2$ case for comparison with earlier sections of the paper.

As discussed previously, the appropriate equations of motion for each particle's worldline are the Yang-Mills-Wong equations in equation (1.1a) and equation (1.1b). We are seeking perturbative solutions, and therefore expand worldline quantities in the coupling:

$$
\begin{aligned}
x_{\alpha}^{\mu}\left(\tau_{\alpha}\right) & =b_{\alpha}^{\mu}+u_{\alpha} \tau_{\alpha}+\Delta^{(1)} x_{\alpha}^{\mu}\left(\tau_{\alpha}\right)+\Delta^{(2)} x_{\alpha}^{\mu}\left(\tau_{\alpha}\right)+\cdots \\
v_{\alpha}^{\mu}\left(\tau_{\alpha}\right) & =u_{\alpha}+\Delta^{(1)} v_{\alpha}^{\mu}\left(\tau_{\alpha}\right)+\Delta^{(2)} v_{\alpha}^{\mu}\left(\tau_{\alpha}\right)+\cdots \\
c_{\alpha}^{a}\left(\tau_{\alpha}\right) & =c_{\alpha}^{a}+\Delta^{(1)} c_{\alpha}^{a}\left(\tau_{\alpha}\right)+\Delta^{(2)} c_{\alpha}^{a}\left(\tau_{\alpha}\right)+\cdots
\end{aligned}
$$

Here $\Delta^{(i)} x_{\alpha}^{\mu}$ indicates quantities entering at $\mathcal{O}\left(g^{2 i}\right)$. Calculating higher order corrections requires solving for the gauge field $A_{\mu}^{a}(x)$, using equation (1.1c). Provided the particle worldlines remain well separated, we can find perturbative solutions using the field equation in the form [11]

$$
\begin{aligned}
\partial^{2} A_{\mu}^{a}(x) & =K_{\mu}^{a}(x), \\
K_{\mu}^{a}(x) & \equiv J_{\mu}^{a}(x)+g f^{a b c} A^{b \nu}(x)\left(\partial_{\nu} A_{\mu}^{c}(x)-F_{\mu \nu}^{c}(x)\right) .
\end{aligned}
$$

The current $K_{\mu}^{a}$ is conserved but gauge dependent; for simplicity we have chosen Lorenz gauge. Writing $\Delta^{(i)} A_{\mu}^{a}(\bar{\ell})$ for perturbative corrections to gauge-field quantities at order $\mathcal{O}\left(g^{2 i-1}\right)$, the LO and NLO gauge fields are solutions to the equations

$$
\begin{aligned}
& \partial^{2}\left[\Delta^{(1)} A_{\mu}^{a}(x)\right]=\Delta^{(1)} J_{\mu}^{a}(x), \\
& \partial^{2}\left[\Delta^{(2)} A_{\mu}^{a}(x)\right]=\Delta^{(2)} J_{\mu}^{a}(x)+g f^{a b c}\left[\Delta^{(1)} A^{b \nu}(x)\left(\partial_{\nu} \Delta^{(1)} A_{\mu}^{c}(x)-\Delta^{(1)} F_{\mu \nu}^{c}(x)\right)\right],
\end{aligned}
$$

respectively - note that the LO equation is the same as for Abelian electrodynamics. We solve by Fourier transforming, ${ }^{9}$ using tildes to represent the Fourier transformed quantity. Solving the LO equation, it is easy to show that the leading order field is

$$
\Delta^{(1)} \tilde{A}^{a \mu}(\bar{\ell})=-g \sum_{\alpha} \hat{\delta}\left(u_{\alpha} \cdot \bar{\ell}\right) e^{i \bar{\ell} \cdot b_{\alpha}} \frac{c_{\alpha}^{a} u_{\alpha}^{\mu}}{\bar{\ell}^{2}}
$$

\footnotetext{
${ }^{9}$ Our conventions for the Fourier transform are $g(x)=\int \hat{\mathrm{d}}^{4} \bar{\ell} e^{-i \bar{\ell} \cdot x} \tilde{g}(\bar{\ell})$ and $\tilde{g}(\bar{\ell})=\int \mathrm{d}^{4} x e^{i \bar{\ell} \cdot x} g(x)$.
} 
It will be useful to define the straight line trajectory $y_{\alpha}^{\mu} \equiv b_{\alpha}^{\mu}+u_{\alpha}^{\mu} \tau_{\alpha}$ corresponding to the initial unperturbed worldlines. With this definition the LO equations of motion become

$$
\begin{aligned}
m_{\alpha} \frac{\mathrm{d}^{2} \Delta^{(1)} x_{\alpha}^{\mu}}{\mathrm{d} \tau_{\alpha}^{2}} & =g c_{\alpha}^{a} \int \hat{\mathrm{d}}^{4} \bar{\ell} e^{-i \bar{\ell} \cdot y_{\alpha}} \Delta^{(1)} \tilde{F}^{a \mu \nu}(\bar{\ell}) u_{\alpha \nu}, \\
\frac{\mathrm{d} \Delta^{(1)} c_{\alpha}^{a}}{\mathrm{~d} \tau_{\alpha}} & =g f^{a b c} \int \hat{\mathrm{d}}^{4} \bar{\ell} e^{-i \bar{\ell} \cdot y_{\alpha}} u_{\alpha}^{\mu} \Delta^{(1)} \tilde{A}_{\mu}^{b}(\bar{\ell}) c_{\alpha}^{c},
\end{aligned}
$$

while at $\mathrm{NLO}, \mathcal{O}\left(g^{4}\right)$, we have

$$
\begin{array}{r}
\frac{\mathrm{d}^{2} \Delta^{(2)} x_{\alpha}^{\mu}}{\mathrm{d} \tau_{\alpha}^{2}}=\frac{g}{m_{\alpha}} \int \hat{\mathrm{d}}^{4} \bar{\ell} e^{-i \bar{\ell} \cdot y_{\alpha}}\left[\Delta^{(1)} \tilde{F}^{a \mu \nu}(\bar{\ell}) \Delta^{(1)} v_{\alpha \nu} c_{\alpha}^{a}+\Delta^{(1)} \tilde{F}^{a \mu \nu}(\bar{\ell})\right. \\
\left.\times u_{\alpha \nu} \Delta^{(1)} c_{\alpha}^{a}+\Delta^{(2)} \tilde{F}^{a \mu \nu}(\bar{\ell}) u_{\alpha \nu} c_{\alpha}^{a}-i \bar{\ell} \cdot \Delta^{(1)} x_{\alpha} \Delta^{(1)} \tilde{F}^{a \mu \nu}(\bar{\ell}) u_{\alpha \nu} c_{\alpha}^{a}\right], \\
\frac{\mathrm{d} \Delta^{(2)} c_{\alpha}^{a}}{\mathrm{~d} \tau_{\alpha}}=g f^{a b c} \int \hat{\mathrm{d}}^{4} \bar{\ell} e^{-i \bar{\ell} \cdot y_{\alpha}}\left[u_{\alpha} \cdot \Delta^{(1)} \tilde{A}^{b}(\bar{\ell}) \Delta^{(1)} c_{\alpha}^{c}+\Delta^{(1)} v_{\alpha} \cdot \Delta^{(1)} A^{b}(\bar{\ell}) c_{\alpha}^{c}\right. \\
\left.+u_{\alpha} \cdot \Delta^{(2)} \tilde{A}^{b} c_{\alpha}^{c}-i \bar{\ell} \cdot \Delta^{(1)} x_{\alpha} u_{\alpha} \cdot \Delta^{(1)} \tilde{A}^{b}(\bar{\ell}) c_{\alpha}^{c}\right] .
\end{array}
$$

These NLO equations involve the LO corrections to the fields and the particles' colours, positions and velocities, so although they are not the main quantities of interest we will need to integrate the expressions in equation (5.5); for example,

$$
\Delta c_{\alpha}^{a}\left(\tau_{\alpha}\right)=\int_{-\infty}^{\tau_{\alpha}} \mathrm{d} \tau_{\alpha}^{\prime} \frac{\mathrm{d} c_{\alpha}^{a}}{\mathrm{~d} \tau_{\alpha}^{\prime}} .
$$

In performing these integrals one must include an $i \epsilon$ convergence factor, so the definition of $y_{\alpha}^{\mu}$ is modified such that $\bar{\ell} \cdot y_{\alpha}=\bar{\ell} \cdot b_{\alpha}+\left(u_{\alpha} \cdot \bar{\ell}+i \epsilon\right) \tau_{\alpha}$. This yields

$$
\begin{aligned}
& \Delta^{(1)} x_{\alpha}^{\mu}\left(\tau_{\alpha}\right)=i g^{2} \sum_{\beta \neq \alpha} \frac{c_{\alpha} \cdot c_{\beta}}{m_{\alpha}} \int \hat{\mathrm{d}}^{4} \bar{\ell} e^{i\left(\bar{\ell} \cdot b_{\beta}-\bar{\ell} \cdot y_{\alpha}\right)} \hat{\delta}\left(\bar{\ell} \cdot u_{\beta}\right) \frac{\bar{\ell} \cdot u_{\alpha} u_{\beta}^{\mu}-\bar{\ell}^{\mu} u_{\alpha} \cdot u_{\beta}}{\overline{\ell^{2}}\left(\bar{\ell} \cdot u_{\alpha}+i \epsilon\right)^{2}}, \\
& \Delta^{(1)} c_{\alpha}^{a}\left(\tau_{\alpha}\right)=i g^{2} \sum_{\beta \neq \alpha} f^{a b c} c_{\alpha}^{b} c_{\beta}^{c} u_{\alpha} \cdot u_{\beta} \int \hat{\mathrm{d}}^{4} \bar{\ell} e^{i\left(\bar{\ell} \cdot b_{\beta}-\bar{\ell} \cdot y_{\alpha}\right)} \frac{\hat{\delta}\left(\bar{\ell} \cdot u_{\beta}\right)}{\bar{\ell}^{2}} \frac{1}{\left(\bar{\ell} \cdot u_{\alpha}+i \epsilon\right)} .
\end{aligned}
$$

We now have the information to determine the NLO field, which is

$$
\begin{aligned}
\Delta^{(2)} \tilde{A}_{\mu}^{a}\left(\bar{\ell}_{1}\right)= & -g^{3} \frac{1}{\bar{\ell}_{1}^{2}} \sum_{\beta \neq \alpha} \int \hat{\mathrm{d}}^{4} \bar{\ell}_{2} e^{i\left(\bar{\ell}_{1}-\bar{\ell}_{2}\right) \cdot b_{\alpha}} e^{i \bar{\ell}_{2} \cdot b_{\beta}} \hat{\delta}\left(\left(\bar{\ell}_{1}-\bar{\ell}_{2}\right) \cdot u_{\alpha}\right) \hat{\delta}\left(\bar{\ell}_{2} \cdot u_{\beta}\right) \\
& \times\left\{\frac{c_{\alpha}^{a} c_{\alpha} \cdot c_{\beta}}{m_{\alpha} \bar{\ell}_{2}^{2}}\left(-\frac{\bar{\ell}_{2}^{\mu} u_{\alpha} \cdot u_{\beta}}{\bar{\ell}_{2} \cdot u_{\alpha}}+\frac{\bar{\ell}_{1} \cdot \bar{\ell}_{2} u_{\alpha} \cdot u_{\beta} u_{\alpha}^{\mu}}{\left(\bar{\ell}_{2} \cdot u_{\alpha}\right)^{2}}-\frac{\bar{\ell}_{1} \cdot u_{\beta} u_{\alpha}^{\mu}}{\bar{\ell}_{2} \cdot u_{\alpha}}+u_{\beta}^{\mu}\right)\right. \\
& \left.+\frac{i f^{a b c} c_{\alpha}^{b} c_{\beta}^{c}}{\bar{\ell}_{2}^{2}}\left(\frac{\bar{\ell}_{2}^{\mu} u_{\alpha} \cdot u_{\beta}}{\left(\bar{\ell}_{1}-\bar{\ell}_{2}\right)^{2}}-\frac{2 \bar{\ell}_{2} \cdot u_{\alpha} u_{\beta}^{\mu}}{\left(\bar{\ell}_{1}-\bar{\ell}_{2}\right)^{2}}+\frac{u_{\alpha} \cdot u_{\beta} u_{\alpha}^{\mu}}{\bar{\ell}_{2} \cdot u_{\alpha}}\right)\right\} .
\end{aligned}
$$

It is now very simple to use the Fourier transform of the Yang-Mills equation in equation (5.2) to calculate the LO momentum space current. In this context it is useful to 
rename the momentum of the field $\bar{k}$, relabel $\bar{\ell}_{2}=\bar{q}_{2}$, and introduce $\bar{q}_{1}=\bar{\ell}_{1}-\bar{\ell}_{2}$. Then we find that

$$
\begin{aligned}
\Delta^{(2)} \tilde{K}^{a \mu}(\bar{k})= & g^{3} \sum_{\beta \neq \alpha} \int \hat{\mathrm{d}}^{4} \bar{q}_{1} \hat{\mathrm{d}}^{4} \bar{q}_{2} \hat{\delta}^{(4)}\left(\bar{k}-\bar{q}_{1}-\bar{q}_{2}\right) \hat{\delta}\left(u_{\alpha} \cdot \bar{q}_{1}\right) \hat{\delta}\left(u_{\beta} \cdot \bar{q}_{2}\right) e^{i b_{\alpha} \cdot \bar{q}_{1}} e^{i b_{\beta} \cdot \bar{q}_{2}} \\
\times & \left\{\frac{c_{\alpha} \cdot c_{\beta}}{\bar{q}_{2}^{2} \bar{k} \cdot u_{\alpha}} \frac{c_{\alpha}^{a}}{m_{\alpha}}\left[-\left(u_{\alpha} \cdot u_{\beta}\right)\left(\bar{q}_{2}^{\mu}-\frac{\bar{k} \cdot \bar{q}_{2}}{\bar{k} \cdot u_{\alpha}} u_{\alpha}^{\mu}\right)+\bar{k} \cdot u_{\alpha} u_{\beta}^{\mu}-\bar{k} \cdot u_{\beta} u_{\alpha}^{\mu}\right]\right. \\
& \left.+\frac{i f^{a b c} c_{\alpha}^{b} c_{\beta}^{c}}{\bar{q}_{1}^{2} \bar{q}_{2}^{2}}\left[2 \bar{k} \cdot u_{\beta} u_{\alpha}^{\mu}-u_{\alpha} \cdot u_{\beta} \bar{q}_{1}^{\mu}+u_{\alpha} \cdot u_{\beta} \frac{\bar{q}_{1}^{2}}{\bar{k} \cdot u_{\alpha}} u_{\alpha}^{\mu}\right]\right\} .
\end{aligned}
$$

This result was first obtained in ref. [11]. Comparing against equation (4.19), we can see that (up to an irrelevant overall sign) the two particle restriction of the current is equal to the $\mathrm{LO}$ radiation kernel calculated using amplitudes. ${ }^{10}$

Returning to the impulse, we can skip over the current and substitute the NLO field into equation (5.6). A straightforward but tedious calculation then yields the results for the NLO corrections given in appendix C. The observable quantities, the impulses, are defined by

$$
\Delta c_{\alpha}^{a} \equiv \int_{-\infty}^{\infty} \mathrm{d} \tau_{\alpha} \frac{\mathrm{d} c_{\alpha}^{a}}{\mathrm{~d} \tau_{\alpha}}, \quad \Delta p_{\alpha}^{\mu} \equiv m_{\alpha} \int_{-\infty}^{\infty} \mathrm{d} \tau_{\alpha} \frac{\mathrm{d} v_{\alpha}^{\mu}}{\mathrm{d} \tau_{\alpha}}
$$

Using the results for $\Delta^{(2)} c_{\alpha}^{a}$ it is straightforward to show, after a redefinition of the integration variables, that the NLO colour impulse takes the form

$$
\begin{aligned}
\Delta c_{\alpha}^{a,(2)}= & f^{a b c} \int \hat{\mathrm{d}}^{4} \bar{q} e^{i b_{2} \cdot \bar{q}} e^{-i b_{1} \cdot \bar{q}} \hat{\delta}\left(\bar{q} \cdot u_{1}\right) \hat{\delta}\left(\bar{q} \cdot u_{2}\right) \int \frac{\hat{\mathrm{d}}^{4} \bar{\ell}}{\bar{\ell}^{2}(\bar{\ell}-\bar{q})^{2}} \\
& \times\left\{\hat{\delta}\left(\bar{\ell} \cdot u_{2}\right)\left[\frac{c_{1} \cdot c_{2} c_{1}^{b} c_{2}^{c}}{m_{1}}\left(1+\frac{\left(u_{1} \cdot u_{2}\right)^{2}\left(\bar{\ell}^{2}-\bar{\ell} \cdot \bar{q}\right)}{\left(\bar{\ell} \cdot u_{1}-i \epsilon\right)^{2}}\right)+i f^{b d e} c_{2}^{c} c_{2}^{d} c_{1}^{e} \overline{\bar{\ell} \cdot u_{1}-i \epsilon}\right]\right. \\
& +\hat{\delta}\left(\bar{\ell} \cdot u_{1}\right)\left[\frac{c_{1} \cdot c_{2} c_{1}^{b} c_{2}^{c}}{m_{2}}\left(1+\frac{\left(u_{1} \cdot u_{2}\right)^{2}\left(\bar{\ell}^{2}-\bar{\ell} \cdot \bar{q}\right)}{\left(\bar{\ell} \cdot u_{2}+i \epsilon\right)^{2}}\right)+i f^{b d e} c_{1}^{c} c_{1}^{d} c_{2}^{e} \frac{\left(u_{1} \cdot u_{2}\right)^{2}}{\bar{\ell} \cdot u_{2}+i \epsilon}\right] \\
& \left.-2 i f^{b d e} c_{1}^{c} c_{1}^{d} c_{2}^{e} \hat{\delta}\left(\bar{\ell} \cdot u_{1}\right) \frac{\bar{\ell} \cdot u_{2}}{\bar{q}^{2}}\right\} .
\end{aligned}
$$

Notice that the signs of the $i \epsilon$ on the second and third lines in the above equation are different. This is a simple consequence of a change of variables required to associate the loop momenta of the classical calculation to that derived from amplitudes (see appendix C). To see that this indeed is the same as our earlier result we must manipulate the $i \epsilon$ factors in the denominators further. For the quadratic denominator we can replace

$$
\frac{1}{\left(\bar{\ell} \cdot u_{\alpha}+i \epsilon\right)^{2}}=i \hat{\delta}^{\prime}\left(\bar{\ell} \cdot u_{\alpha}\right)+\frac{1}{\left(\bar{\ell} \cdot u_{\alpha}-i \epsilon\right)^{2}},
$$

and for the linear denominator we make the shift $\bar{\ell} \rightarrow \bar{q}-\bar{\ell}$, which simply has the effect of changing the sign of the $i \epsilon .{ }^{11}$ Then these terms can be averaged and combined to form a

\footnotetext{
${ }^{10}$ Notice that we set $b_{2}=0$ in sections 3 and 4 using translation symmetry.

${ }^{11}$ The $\bar{q} \cdot u_{\alpha}$ term can be ignored due to the delta function $\delta\left(\bar{q} \cdot u_{\alpha}\right)$.
} 
delta function. These procedures are the same as for the momentum impulse [15] and are briefly reviewed in appendix B. After these manipulations, the result from amplitudes in equation (3.47) matches the colour impulse computed classically in equation (5.12), up to the term on the last line.

This final term is spurious, and can be traced back to the non-Abelian correction to the gauge field at NLO, shown in the bottom line of equation (5.9). We observe that the term is proportional to the integral

$$
u_{2} \cdot I=\int \hat{\mathrm{d}}^{4} \bar{\ell} \hat{\delta}\left(\bar{\ell} \cdot u_{1}\right) \frac{\bar{\ell} \cdot u_{2}}{\bar{\ell}^{2}(\bar{\ell}-\bar{q})^{2}}
$$

which vanishes on the support of $\hat{\delta}\left(\bar{q} \cdot u_{1}\right)$ and $\hat{\delta}\left(\bar{q} \cdot u_{2}\right)$. This can easily be seen by writing ${ }^{12}$ $I^{\mu}=A u_{1}^{\mu}+B \bar{q}^{\mu}$; then we have that $A=0$, and hence $u_{2} \cdot I=0$.

The momentum impulse follows through similarly, and for the case of two particles we find that the result is the same as in Abelian electrodynamics, calculated by KMOC [15], but with the replacement $Q_{1} Q_{2} \rightarrow c_{1} \cdot c_{2}$. This is in agreement with the quantum calculation of section 3.4.

\subsection{Total radiated colour and asymptotic symmetries}

Our classical calculation of the NLO impulse relied on the order $g^{3}$ gauge field in equation (5.9). This gauge field in isolation is also of interest, because it is the leading radiation field generated by the scattering of the two particles. It therefore describes the transport of momentum and colour by the classical YM field itself. This classical transport of colour deserves more discussion.

The Noether current for a vector field transforming in the adjoint of the colour group is

$$
j_{\mu}^{a}(x)=-f^{a b c} A^{b \nu}(x)\left(\partial_{\mu} A_{\nu}^{c}(x)-\partial_{\nu} A_{\mu}^{c}(x)\right) .
$$

To measure the instantaneous rate of colour radiation at a time $t$ during a scattering event, we surround the particles by a large sphere and measure the flux of $j_{\mu}^{a}$ across its surface, taking the limit that the radius of the sphere goes to infinity. More specifically, we are interested in outgoing radiation from our scattering event, so we take this large radius limit at fixed retarded time $u=t-r$. We then integrate over all retarded times. Thus the surface of integration - namely the null future boundary $\mathscr{I}^{+}$of Minkowski space - is three-dimensional, parameterised by $u$ and the coordinates on the two-sphere. The colour radiated to $\mathscr{I}^{+}$is ${ }^{13}$

$$
\begin{aligned}
R_{\mathrm{col}}^{a} & =\int_{\mathscr{I}+} * j^{a} \\
& =-\int_{-\infty}^{\infty} \mathrm{d} u \lim _{r \rightarrow \infty} \int \mathrm{d} \Omega_{2} r^{2} j_{r}^{a},
\end{aligned}
$$

where $j^{a}=j_{\mu}^{a} \mathrm{~d} x^{\mu}$ and $* j^{a}$ is its Hodge dual.

\footnotetext{
${ }^{12}$ Any additional vectorial dependence arising by regulating the divergent integral will have vanishing dot product with $u_{2}$.

${ }^{13}$ We use Bondi coordinates $u, r, \theta$ and $\phi$.
} 
To evaluate this integral, we need an expression for the asymptotic field. This satisfies the Yang-Mills equation, given in linearised form in equation (5.2). Since there is no incoming radiation in our situation, we impose retarded boundary conditions. Using the standard large-distance expansion of the retarded Green's function we readily find

$$
A_{\mu}^{a}(x)=\left.\frac{1}{4 \pi r} \int \frac{\mathrm{d} \omega}{2 \pi} e^{-i \omega u} \tilde{K}_{\mu}^{a}(\bar{k})\right|_{\bar{k}^{\nu}=(\omega, \omega \hat{\boldsymbol{x}})}+\mathcal{O}\left(\frac{1}{r^{2}}\right),
$$

where $r=|\boldsymbol{x}|$ and $t=x^{0}$. It may also help the reader to record the derivative of the field, which is

$$
\partial_{\mu} A_{\nu}^{a}(x)=-\left.\frac{i}{4 \pi r} \int \frac{\mathrm{d} \omega}{2 \pi} \bar{k}_{\mu} \tilde{K}_{\nu}^{a}(\bar{k}) e^{-i \omega u}\right|_{\bar{k}^{\rho}=(\omega, \omega \hat{\boldsymbol{x}})}+\mathcal{O}\left(\frac{1}{r^{2}}\right)
$$

Hence upon integrating over delta functions the total radiated charge is

$$
R_{\mathrm{col}}^{a}=\left.\frac{i}{(4 \pi)^{2}} \int_{-\infty}^{\infty} \frac{\mathrm{d} \omega}{2 \pi} \int \mathrm{d} \Omega_{2} f^{a b c} \tilde{K}^{b \nu}(-\bar{k})\left(k_{\nu} \tilde{K}_{r}^{c}(k)-\bar{k}_{r} \tilde{K}_{\nu}^{c}(\bar{k})\right)\right|_{\bar{k}=(\omega, \omega \hat{\boldsymbol{x}})} .
$$

This expression can be considerably simplified. Current conservation is $k^{\nu} \tilde{K}_{\nu}^{a}(k)=0$, so the first term in parentheses vanishes. In the second term, we have $k_{r}=-\omega$. We can further exploit the symmetry of the integral, and reality of the current $K_{\mu}^{a}(x)$ to show

$$
\begin{aligned}
R_{\text {col }}^{a} & =\left.\frac{i}{(2 \pi)^{3}} \int_{0}^{\infty} \mathrm{d} \omega \omega^{2} \int \mathrm{d} \Omega_{2} \frac{1}{2 \omega} f^{a b c} \tilde{K}_{\nu}^{b}(-\bar{k}) \tilde{K}^{c \nu}(\bar{k})\right|_{\bar{k}=(\omega, \omega \hat{\boldsymbol{x}})} \\
& =\frac{i}{(2 \pi)^{3}} \int \mathrm{d}^{3} \bar{k} \mathrm{~d} \bar{k}^{0} \frac{\delta\left(\bar{k}^{0}-|\overline{\boldsymbol{k}}|\right)}{2|\overline{\boldsymbol{k}}|} f^{a b c} \tilde{K}_{\nu}^{b}(-\bar{k}) \tilde{K}^{c \nu}(\bar{k}) \\
& =\int \mathrm{d} \Phi(\bar{k}) i f^{a b c} \tilde{K}_{\nu}^{b *}(\bar{k}) \tilde{K}^{c \nu}(\bar{k}) .
\end{aligned}
$$

Finally, we use completeness of the polarisation vectors to write the classical total radiated colour in precisely the same form as the quantum expression:

$$
R_{\mathrm{col}}^{a}=-i f^{a b c} \sum_{\sigma= \pm} \int \mathrm{d} \Phi(\bar{k})\left(\varepsilon_{\sigma}^{*} \cdot \tilde{K}^{*}(\bar{k})\right)^{b}\left(\varepsilon_{\sigma} \cdot \tilde{K}(\bar{k})\right)^{c} .
$$

Comparing with equation (4.8) confirms that, in the classical limit, the radiation kernel coincides (up to a possible sign) with $\varepsilon_{\sigma} \cdot \tilde{K}^{a}(\bar{k})$ at large distances.

Let us finish with a few additional remarks on this radiated colour. In ordinary electrodynamics it is elementary that charge is connected with the current appearing in the equation of motion. Although we made use of the Noether current in our discussion above, it remains the case that the radiated charge is connected to the current $K^{a}$ in the linearised form of equation (5.2). It is easy to check, using the explicit asymptotic field of equation (5.17), that the radiated charge is

$$
R_{\mathrm{col}}^{a}=\frac{1}{g} \int_{\mathscr{I}^{+}} * K^{a} .
$$

We may now make use of equation (5.2) in the form

$$
\mathrm{d} * F^{a}=-* K^{a}
$$


to write

$$
R_{\mathrm{col}}^{a}=-\frac{1}{g} \int_{\mathscr{I}^{+}} \mathrm{d} * F^{a},
$$

where $F^{a}$ is the linearised field strength. The radiated charge may therefore also be reconstructed by integration over the boundaries $\mathscr{I}_{ \pm}^{+}$of $\mathscr{I}^{+}$as

$$
g R_{\mathrm{col}}^{a}=\int_{\mathscr{I}_{-}^{+}} * F^{a}-\int_{\mathscr{I}_{+}^{+}} * F^{a}=g c_{\text {initial }}-g c_{\text {final }} .
$$

In other words, the radiated charge is the difference between initial and final charges, as measured by integrating the electric fields over large spheres in the far past and the far future: total colour charge is conserved, as we also saw using quantum mechanical methods in equation (4.4).

Although our focus was on radiation of global charge, some of the expressions above are also relevant in the discussion of the larger asymptotic symmetry group of Yang-Mills theory, see for example $[106-110,112,137,138]$. It would be interesting to broaden our analysis to this context, particularly in the context of the infrared structure of loop amplitudes.

\section{Discussion}

In this article, we developed methods for computing classical observables in Yang-Mills theories from scattering amplitudes. This amounts to an extension of the scope of the KMOC formalism [15] to encompass perturbative Yang-Mills theory. In addition to the observables familiar from electrodynamics and gravity, namely the momentum impulse and the total radiated momentum, we constructed two new observables: the colour impulse and total radiated colour charge.

Our underlying motivation is to understand the dynamics of classical general relativity through the double copy. In particular, we are interested in the relativistic two-body problem which is so central to the physics of the compact binary coalescence events observed by LIGO and Virgo. Consequently, we focused on observables in two-body events. Although we only considered unbound (scattering) events, it is possible to determine the physics of bound states from our observables. This can be done concretely using effective theories [14]. We also hope that it may be possible to connect our observables more directly to bound states using analytic continuation, in a manner similar to the work of Kälin and Porto [32, 34].

The emergence of the classical theory from an underlying perturbative quantum field theory is surprisingly intricate. Coherent states play an important role in this story, as emphasised by Yaffe [124] in the context of large $N$ theories. In section 2 we emphasised the role of coherent states in describing the colour structure of particles in the classical approximation. It is also important that the representation of the corresponding quantum field is large. This is in exact analogy with the emergence of a classical spin from a quantum system, and indeed the states we used for colour can equally be used to describe spin. Furthermore the physics of the colour impulse in YM theory is closely analogous to the physics of angular momentum and the associated angular impulse [95, 96]. Since the story for colour is a little simpler, we expect it to be a useful toy model for spin in gravity. 
We studied the impulse and its colourful counterpart at NLO in YM theory. One surprise in our work was that the part of the (four-point) amplitude which is relevant in the classical theory is exactly proportional to the classical part of the QED four-point amplitude. Indeed the impulse at next-to-leading order in the YM case is basically equal to the QED case; the only difference is a charge-to-colour replacement. This is a little peculiar because it is natural to expect the non-linearity of the Yang-Mills field to enter at this order (and it does so in the quantum theory). Nevertheless the colour impulse, which is intrinsically non-Abelian by definition, is non-vanishing. Although it is constructed from the same one-loop amplitude, an interplay of colour commutators and classically singular terms in the amplitude results in an expression for the colour impulse which involves various different colour factors. We confirmed the results of our calculations by a direct classical computation using the Yang-Mills-Wong worldline formalism. It is interesting to compare our methods to those of Shen [85], who implemented the double copy at NLO wholly within the classical worldline formalism following ground-breaking work of Goldberger and Ridgway [11]. Shen found it necessary to include vanishing terms involving structure constants in his work. Similarly, in our context, some colour factors are paired with kinematic numerators proportional to $\hbar$. It would be interesting to use the tools developed in this paper to explore the double copy construction of Shen [85] from the perspective of amplitudes.

Throughout our paper, we emphasised that scattering amplitudes can be used to determine classical YM observables. But so do Wong's equations. We have not addressed the question of whether it is easier to find a particular observable from amplitudes or from the Wong equations. This question isn't really of interest to us since our goal is to understand gravity, where amplitudes are much easier to compute than any (known) classical procedure. But possibly our work offers a way to combine the advantages of classical equations and the double copy. We provided explicit expressions for YM observables in terms of amplitudes; given a determination of these observables from the Wong equations, then it is possible to solve for the (classical part of the) amplitude. If it is possible to compute a corresponding gravitational amplitude from the double copy unambiguously from the classical parts of a Yang-Mills amplitude, then this method would allow for the computation of observables in gravity from the Wong equations. Compared to the worldline double copy of Goldberger, Ridgway [11] and Shen [85] this suggestion would implement the double copy in a more standard manner. Our methods may also shine light on the difficulty implementing the double copy off-shell in the worldline theory discussed in [87], since one could check proposals for implementing a worldline double copy against our formulae.

Our expression for the colour impulse is in many ways similar to the KMOC expression for the ordinary impulse. In essence the impulse describes a transfer of a small amount of momentum $\hbar \bar{q}$, weighted by an amplitude of order $1 / \hbar$. Thus the momentum transferred by many gluons leads to a macroscopic impulse. In the colour case, the small momentum transferred is replaced by a colour commutator. This is reminiscent of the transition from fuzzy spaces to the continuum (see, for example $[139,140]$ ): the momentum transfer in the impulse is the Fourier transform of a derivative, corresponding to the commutator in a fuzzy space. Perhaps there is a clue here to how the double copy works. 
Turning to radiation of colour, a first comment is that the relevant amplitude is no longer proportional to the QED case. This means that at NNLO the impulse will no longer be proportional to the QED impulse, because the radiated momenta are genuinely different in the two cases. One motivation for studying impulse and radiation together is that they are related by conservation of momentum, so the five-point radiation terms capture dissipative effects in the impulse. The physics of momentum conservation and dissipation is rich, so we look forward to further work in this area.

Colour radiation is also interesting from the point of view of asymptotic symmetry groups. Yang-Mills theory is an interesting toy model for gravity in this context, as pointed out in an early paper by Lüscher [137]. It would be very interesting to study colour radiation at NLO, in particular to understand what becomes of the infrared divergences of the loop amplitudes, and their impact on soft theorems. The Yang-Mills case is particularly subtle in view of the presence of collinear divergences. In electromagnetism and gravity a first step in these directions has recently been made [46], where the connection between quantum and classical soft theorems in electromagnetism and gravity was studied using radiation kernels. We look forward to future progress on these fronts.

\section{Acknowledgments}

We thank Roger Horsley, David Kosower, Seán Mee, Alexander Ochirov and Siddharth Pandey for useful discussions, and Ingrid Holm for correcting some typos in our equations. BM and AR are supported by STFC studentships ST/R504737/1 and ST/T506060/1 respectively. LDLC and DOC are supported by the STFC grant ST/P0000630/1. This research was supported by the Munich Institute for Astro- and Particle Physics (MIAPP) which is funded by the Deutsche Forschungsgemeinschaft (DFG, German Research Foundation) under Germany's Excellence Strategy - EXC-2094 - 390783311. Some of our figures were produced with the help of TikZ-Feynman [141].

\section{A Charge factorisation in $\mathrm{SU}(3)$}

In this appendix we prove equation (2.34). Using the coherent states restricted to the $\mathrm{SU}(3)$ irreducible representation $\left[n_{1}, n_{2}\right]$ in equation (2.29), we have

$$
\begin{aligned}
\left\langle\xi \zeta\left|\mathbb{C}^{a} \mathbb{C}^{b}\right| \xi \zeta\right\rangle_{\left[n_{1}, n_{2}\right]}= & \frac{1}{\left(n_{1} ! n_{2} !\right)}\langle 0|\left(\zeta^{*} \cdot b\right)^{n_{2}}\left(\xi^{*} \cdot a\right)^{n_{1}}\left(a^{\dagger} \lambda^{a} a-b^{\dagger} \bar{\lambda}^{a} b\right) \\
& \times\left(a^{\dagger} \lambda^{b} a-b^{\dagger} \bar{\lambda}^{b} b\right)\left(\zeta \cdot b^{\dagger}\right)^{n_{2}}\left(\xi \cdot a^{\dagger}\right)^{n_{1}}|0\rangle \\
= & \frac{1}{\left(n_{1} ! n_{2} !\right)}\langle 0|\left(\zeta^{*} \cdot b\right)^{n_{2}}\left(\xi^{*} \cdot a\right)^{n_{1}}\left(a^{\dagger} \lambda^{a} a a^{\dagger} \bar{\lambda}^{b} a-b^{\dagger} \bar{\lambda}^{a} b a^{\dagger} \lambda^{b} a\right. \\
& \left.-a^{\dagger} \lambda^{a} a b^{\dagger} \bar{\lambda}^{b} b-b^{\dagger} \bar{\lambda}^{a} b b^{\dagger} \bar{\lambda}^{b} b\right)\left(\zeta \cdot b^{\dagger}\right)^{n_{2}}\left(\xi \cdot a^{\dagger}\right)^{n_{1}}|0\rangle .
\end{aligned}
$$


Note that we can consider the $a$ and $b$ terms separately since they commute. The terms with only two $a$ operators (or $b$ 's) reduce to products of the form

$$
\begin{aligned}
\left\langle\xi \zeta\left|a^{\dagger i} a_{j}\right| \xi \zeta\right\rangle_{\left[n_{1}, n_{2}\right]} & =\frac{1}{\left(n_{1} ! n_{2} !\right)}\left\langle 0\left|\left(\zeta^{*} \cdot b\right)^{n_{2}}\left(\xi^{*} \cdot a\right)^{n_{1}} a^{\dagger i} a_{j}\left(\zeta \cdot b^{\dagger}\right)^{n_{2}}\left(\xi \cdot a^{\dagger}\right)^{n_{1}}\right| 0\right\rangle \\
& =n_{1} \xi^{* i} \xi_{j},
\end{aligned}
$$

and similarly for expressions involving $b^{i} b_{j}^{\dagger}$. We made use of the fact that the states are normalised. Next we have the term involving four $a$ 's (or $b$ 's), which yields

$$
\left\langle\xi \zeta\left|a^{\dagger i} a_{j} a^{\dagger k} a_{l}\right| \xi \zeta\right\rangle_{\left[n_{1}, n_{2}\right]}=n_{1} \xi^{* i} \xi_{l} \delta_{j}^{k}+n_{1}\left(n_{1}-1\right) \xi^{* i} \xi_{l} \xi^{* k} \xi_{j} .
$$

In the limit where $n_{1}$ is large we can replace ${ }^{14}$ the $n(n-1)$ factor with $n^{2}$. Using this and returning all $\lambda$ and $\hbar$ factors we find

$$
\hbar^{2}\left(\lambda^{a}\right)_{i}{ }^{j}\left(\lambda^{b}\right)_{k}{ }^{l}\left\langle\xi \zeta\left|a^{\dagger i} a_{j} a^{\dagger k} a_{l}\right| \xi \zeta\right\rangle_{\left[n_{1}, n_{2}\right]}=\hbar^{2} n_{1}^{2} \xi^{*} \lambda^{a} \xi \xi^{*} \lambda^{b} \xi+\hbar^{2} n_{1} \xi^{*} \lambda^{a} \cdot \lambda^{b} \xi .
$$

Now, gathering all the terms with a pair of $a$ 's and a pair of b's, which are simply products of the expressions in equation (A.2) contracted with Gell-Mann matrices, we have that

$$
\begin{aligned}
\left\langle\xi \zeta\left|\mathbb{C}^{a} \mathbb{C}^{b}\right| \xi \zeta\right\rangle_{\left[n_{1}, n_{2}\right]}= & \hbar^{2}\left(n_{1}^{2} \xi^{*} \lambda^{a} \xi \xi^{*} \lambda^{b} \xi+n_{2}^{2} \zeta^{*} \bar{\lambda}^{a} \zeta \zeta^{*} \bar{\lambda}^{b} \zeta-n_{1} n_{2} \xi^{*} \lambda^{a} \xi \zeta^{*} \bar{\lambda}^{b} \zeta\right. \\
& \left.-n_{1} n_{2} \xi^{*} \lambda^{b} \xi \zeta^{*} \bar{\lambda}^{a} \zeta\right)+\hbar\left(\hbar n_{1} \xi^{*} \lambda^{a} \cdot \lambda^{b} \xi-\hbar n_{2} \zeta^{*} \bar{\lambda}^{a} \cdot \bar{\lambda}^{b} \zeta\right) .
\end{aligned}
$$

Recognising the charge expectation values $\left\langle\xi \zeta\left|\mathbb{C}^{a} \mathbb{C}^{b}\right| \xi \zeta\right\rangle_{\left[n_{1}, n_{2}\right]}$ from equation (2.33), this can be written as

$$
\begin{aligned}
\left\langle\xi \zeta\left|\mathbb{C}^{a} \mathbb{C}^{b}\right| \xi \zeta\right\rangle_{\left[n_{1}, n_{2}\right]}= & \left\langle\xi \zeta\left|\mathbb{C}^{a}\right| \xi \zeta\right\rangle_{\left[n_{1}, n_{2}\right]}\left\langle\xi \zeta\left|\mathbb{C}^{b}\right| \xi \zeta\right\rangle_{\left[n_{1}, n_{2}\right]} \\
& +\hbar\left(\hbar n_{1} \xi^{*} \lambda^{a} \cdot \lambda^{b} \xi-\hbar n_{2} \zeta^{*} \bar{\lambda}^{a} \cdot \bar{\lambda}^{b} \zeta\right) .
\end{aligned}
$$

The finite quantity in the classical limit $\hbar \rightarrow 0, n_{i} \rightarrow \infty$ is the product $\hbar n_{i}$. The term inside the brackets on the second line is itself finite, but comes with a lone $\hbar$ coefficient, and thus vanishes in the classical limit. This then proves the factorisation property in equation (2.34).

\section{B Diagrams and amplitude expressions}

In this appendix we gather the expressions necessary for calculating the 1-loop partial amplitudes introduced in section 3.3. We are only interested in the leading classical terms of the relevant topologies, and will not list quantum corrections.

From equation (3.25) we know that the relevant 1-loop topologies for NLO observables in YM theory are those which contributed to the analogous QED calculation in [15]. The QED amplitude

$$
\mathcal{A}^{(1, \mathrm{QED})}=B+C+T_{12}+T_{21}
$$

\footnotetext{
${ }^{14}$ The correction term vanishes in the classical $\hbar \rightarrow 0$ limit regardless.
} 
is constructed from the triangle $T_{i j}$, box $B$, and cross box $C$ diagrams. Beginning with the triangles, the leading classical terms are

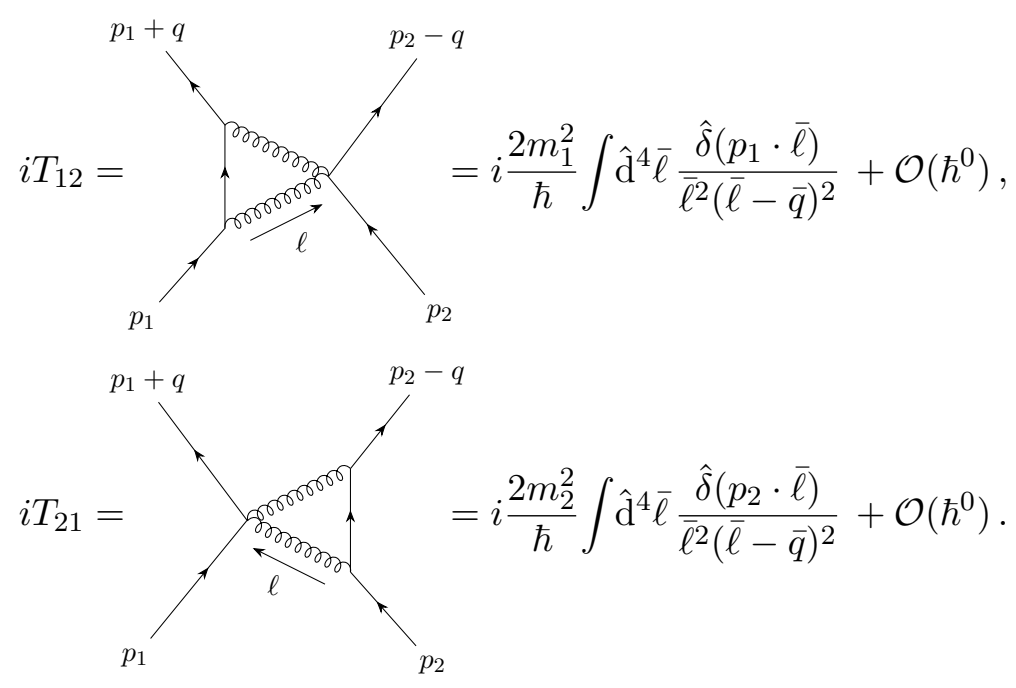

We refer the curious reader to ref. [15] for the detailed calculations: heuristically, the $\hbar$ expansion of the diagrams is conducted by rescaling $\ell \rightarrow \hbar \bar{\ell}$ and $q \rightarrow \hbar \bar{q}$ on the support of the delta functions in equation (3.9). Propagator denominators are expanded as a series in $\hbar$. Noting that the loop integrals are symmetric under the replacement $\bar{\ell} \rightarrow \bar{q}-\bar{\ell}$, this change of variables can be exploited to change the sign of the (Feynman) $i \epsilon$ in massive propagators. Then, averaging over the two expressions for the integral and applying the identities

$$
\begin{aligned}
i \hat{\delta}(x) & =\frac{1}{x-i \epsilon}-\frac{1}{x+i \epsilon} \\
-i \hat{\delta}^{\prime}(x) & =\frac{1}{(x-i \epsilon)^{2}}-\frac{1}{(x+i \epsilon)^{2}}
\end{aligned}
$$

leads to the expressions in equation (B.2). This symmetrisation trick is unnecessary for calculating the individual terms from box topologies, for which we choose the following momentum routing:
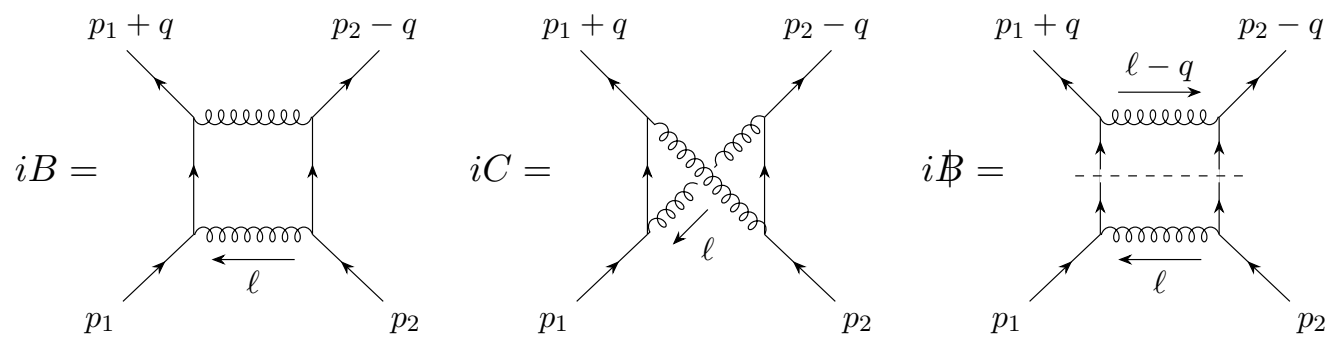

The cut box appears outside of $\mathcal{A}^{(1, \mathrm{QED})}$, forming the quadratic part of the NLO colour kernel, and is defined in equation (3.30). However, these diagrams all have kinematic coefficients of the form

$$
D=D_{-1}+D_{0}+\mathcal{O}\left(\hbar^{0}\right),
$$

where $D_{-1} \sim \mathcal{O}\left(\hbar^{-2}\right)$ and $D_{0} \sim \mathcal{O}\left(\hbar^{-1}\right)$. We choose to label the terms like this as the $\mathcal{O}\left(\hbar^{-1}\right)$ terms are those which generally contribute classically, and we ignore the $\mathcal{O}\left(\hbar^{0}\right)$ 
terms as they always act as quantum corrections. The $\mathcal{O}\left(\hbar^{-2}\right)$ terms would give rise to contributions to the impulse which are classically singular, and as shown in section 3.3 it is necessary to consider all three diagrams in order to see that they cancel. We have

$$
\begin{aligned}
& B_{-1}=i \frac{4\left(p_{1} \cdot p_{2}\right)^{2}}{\hbar^{2}} \int \hat{\mathrm{d}}^{4} \bar{\ell} \frac{1}{\bar{\ell}^{2}(\bar{q}-\bar{\ell})^{2}\left(p_{2} \cdot \bar{\ell}-i \epsilon\right)\left(p_{1} \cdot \bar{\ell}+i \epsilon\right)}, \\
& C_{-1}=-i \frac{4\left(p_{1} \cdot p_{2}\right)^{2}}{\hbar^{2}} \int \hat{\mathrm{d}}^{4} \bar{\ell} \frac{1}{\bar{\ell}^{2}(\bar{q}-\bar{\ell})^{2}} \frac{1}{\left(p_{2} \cdot \bar{\ell}+i \epsilon\right)\left(p_{1} \cdot \bar{\ell}+i \epsilon\right)}, \\
& B_{-1}=-i \frac{4\left(p_{1} \cdot p_{2}\right)^{2}}{\hbar^{2}} \int \hat{\mathrm{d}}^{4} \overline{\hat{\ell}} \frac{\hat{\delta}\left(p_{1} \cdot \bar{\ell}\right) \hat{\delta}\left(p_{2} \cdot \bar{\ell}\right)}{\bar{\ell}^{2}(\bar{q}-\bar{\ell})^{2}},
\end{aligned}
$$

and

$$
\begin{aligned}
B_{0}= & i \frac{2 p_{1} \cdot p_{2}}{\hbar} \int \hat{\mathrm{d}}^{4} \bar{\ell} \frac{1}{\bar{\ell}^{2}(\bar{q}-\bar{\ell})^{2}\left(p_{1} \cdot \bar{\ell}+i \epsilon\right)\left(p_{2} \cdot \bar{\ell}-i \epsilon\right)} \\
& \times\left\{2\left(p_{2}-p_{1}\right) \cdot \bar{\ell}+\left(p_{1} \cdot p_{2}\right) \bar{\ell}^{2}\left(\frac{1}{\left(p_{2} \cdot \bar{\ell}-i \epsilon\right)}-\frac{1}{\left(p_{1} \cdot \bar{\ell}+i \epsilon\right)}\right)\right\}, \\
C_{0}= & -i \frac{2 p_{1} \cdot p_{2}}{\hbar} \int \hat{\mathrm{d}}^{4} \bar{\ell} \overline{\bar{\ell}^{2}(\bar{q}-\bar{\ell})^{2}\left(p_{1} \cdot \bar{\ell}+i \epsilon\right)\left(p_{2} \cdot \bar{\ell}+i \epsilon\right)} \\
& \times\left\{2\left(p_{1}+p_{2}\right) \cdot \bar{\ell}-\left(p_{1} \cdot p_{2}\right)\left(\frac{\bar{\ell}^{2}}{\left(p_{1} \cdot \bar{\ell}+i \epsilon\right)}+\frac{\bar{\ell}^{2}-2 \bar{q} \cdot \bar{\ell}}{\left(p_{2} \cdot \bar{\ell}+i \epsilon\right)}\right)\right\}, \\
B_{0}= & i \frac{2\left(p_{1} \cdot p_{2}\right)^{2}}{\hbar} \int \hat{\mathrm{d}}^{4} \bar{\ell} \frac{1}{\bar{\ell}^{2}(\bar{q}-\bar{\ell})^{2}} \bar{\ell}^{2}\left\{\hat{\delta}\left(p_{1} \cdot \bar{\ell}\right) \hat{\delta}^{\prime}\left(p_{2} \cdot \bar{\ell}\right)-\hat{\delta}^{\prime}\left(p_{1} \cdot \bar{\ell}\right) \hat{\delta}\left(p_{2} \cdot \bar{\ell}\right)\right\} .
\end{aligned}
$$

Here the delta functions in $B$ originate in its definition as the quadratic part of the colour kernel in equation (3.19). Applying the symmetrisation trick and equation (B.3) ensures that the sums of the box and cross box contributions can also be recast in terms of delta functions - hence, upon including the triangle contributions, the leading terms in the expansion of the QED amplitude in equation (B.1) are

$$
\begin{aligned}
\mathcal{A}_{-1}^{(1, \mathrm{QED})}= & i \frac{2\left(p_{1} \cdot p_{2}\right)^{2}}{\hbar^{2}} \int \hat{\mathrm{d}}^{4} \bar{\ell} \frac{1}{\bar{\ell}^{2}(\bar{q}-\bar{\ell})^{2}} \hat{\delta}\left(p_{1} \cdot \bar{\ell}\right) \hat{\delta}\left(p_{2} \cdot \bar{\ell}\right) \\
\mathcal{A}_{0}^{(1, \mathrm{QED})}= & \frac{2}{\hbar} \int \hat{\mathrm{d}}^{4} \bar{\ell} \frac{1}{\bar{\ell}^{2}(\bar{q}-\bar{\ell})^{2}}\left\{i ( p _ { 1 } \cdot p _ { 2 } ) ^ { 2 } \left[\frac { 1 } { 2 } ( \overline { \ell } ^ { 2 } - 2 \overline { \ell } \cdot \overline { q } ) \left(\hat{\delta}\left(p_{1} \cdot \bar{\ell}\right) \hat{\delta}^{\prime}\left(p_{2} \cdot \bar{\ell}\right)\right.\right.\right. \\
& \left.\left.-\hat{\delta}^{\prime}\left(p_{1} \cdot \bar{\ell}\right) \hat{\delta}\left(p_{2} \cdot \bar{\ell}\right)\right)-\left(\bar{\ell}^{2}-\bar{\ell} \cdot \bar{q}\right)\left(\frac{i \hat{\delta}\left(p_{1} \cdot \bar{\ell}\right)}{\left(p_{2} \cdot \bar{\ell}-i \epsilon\right)^{2}}+\frac{i \hat{\delta}\left(p_{2} \cdot \bar{\ell}\right)}{\left(p_{1} \cdot \bar{\ell}+i \epsilon\right)^{2}}\right)\right] \\
& \left.+m_{1}^{2} \hat{\delta}\left(p_{1} \cdot \bar{\ell}\right)+m_{2}^{2} \hat{\delta}\left(p_{2} \cdot \bar{\ell}\right)\right\} .
\end{aligned}
$$

A similar averaging procedure can also be applied to the cut box, yielding

$$
\beta_{0}=-i \frac{2\left(p_{1} \cdot p_{2}\right)^{2}}{\hbar} \int \hat{\mathrm{d}}^{4} \bar{\ell} \frac{\bar{\ell} \cdot \bar{q}}{\bar{\ell}^{2}(\bar{q}-\bar{\ell})^{2}}\left\{\hat{\delta}\left(p_{1} \cdot \bar{\ell}\right) \hat{\delta}^{\prime}\left(p_{2} \cdot \bar{\ell}\right)-\hat{\delta}^{\prime}\left(p_{1} \cdot \bar{\ell}\right) \hat{\delta}\left(p_{2} \cdot \bar{\ell}\right)\right\}
$$

which is the result listed in equation (3.30). 
Finally, for the momentum impulse the cut box $B^{\mu}$ is dressed by a power of the loop momentum, and thus

$$
\begin{aligned}
B_{-1}^{\mu} & =-i \frac{4\left(p_{1} \cdot p_{2}\right)^{2}}{\hbar^{2}} \int \hat{\mathrm{d}}^{4} \bar{\ell} \frac{\bar{\ell}^{\mu} \hat{\delta}\left(p_{1} \cdot \bar{\ell}\right) \hat{\delta}\left(p_{2} \cdot \bar{\ell}\right)}{\bar{\ell}^{2}(\bar{q}-\bar{\ell})^{2}} \\
B_{0}^{\mu} & =i \frac{2\left(p_{1} \cdot p_{2}\right)^{2}}{\hbar} \int \hat{\mathrm{d}}^{4} \bar{\ell} \frac{\bar{\ell}^{\mu}}{\bar{\ell}^{2}(\bar{q}-\bar{\ell})^{2}}\left\{\bar{\ell}^{2} \hat{\delta}\left(p_{1} \cdot \bar{\ell}\right) \hat{\delta}^{\prime}\left(p_{2} \cdot \bar{\ell}\right)-\overline{\ell^{2}} \hat{\delta}\left(p_{2} \cdot \bar{\ell}\right) \hat{\delta}^{\prime}\left(p_{1} \cdot \bar{\ell}\right)\right\} .
\end{aligned}
$$

Shifting with $\bar{\ell} \rightarrow \bar{q}-\bar{\ell}$ and averaging the two expressions, these can be written equivalently as

$$
\begin{aligned}
& B_{-1}^{\mu}=-i \frac{2 g^{4}\left(p_{1} \cdot p_{2}\right)^{2}}{\hbar^{2}} \int \hat{\mathrm{d}}^{4} \bar{\ell} \frac{\bar{q}^{\mu}}{\bar{\ell}^{2}(\bar{q}-\bar{\ell})^{2}} \hat{\delta}\left(p_{1} \cdot \bar{\ell}\right) \hat{\delta}\left(p_{2} \cdot \bar{\ell}\right), \\
& B_{0}^{\mu}=i \frac{2\left(p_{1} \cdot p_{2}\right)^{2}}{\hbar} \int \hat{\mathrm{d}}^{4} \bar{\ell} \frac{\bar{\ell} \cdot(\bar{\ell}-\bar{q})}{\bar{\ell}^{2}(\bar{q}-\bar{\ell})^{2}} \bar{\ell}^{\mu}\left(\hat{\delta}\left(p_{1} \cdot \bar{\ell}\right) \hat{\delta}^{\prime}\left(p_{2} \cdot \bar{\ell}\right)-\hat{\delta}^{\prime}\left(p_{1} \cdot \bar{\ell}\right) \hat{\delta}\left(p_{2} \cdot \bar{\ell}\right)\right) \\
& -i \frac{\left(p_{1} \cdot p_{2}\right)^{2}}{\hbar} \bar{q}^{\mu} \int \hat{\mathrm{d}}^{4} \bar{\ell} \frac{\left(\bar{\ell}^{2}-2 \bar{q} \cdot \bar{\ell}\right)}{\bar{\ell}^{2}(\bar{q}-\bar{\ell})^{2}}\left(\hat{\delta}\left(p_{1} \cdot \bar{\ell}\right) \hat{\delta}^{\prime}\left(p_{2} \cdot \bar{\ell}\right)-\hat{\delta}^{\prime}\left(p_{1} \cdot \bar{\ell}\right) \hat{\delta}\left(p_{2} \cdot \bar{\ell}\right)\right) .
\end{aligned}
$$

Applying the analysis of [15], one would expect the non-Abelian triangles
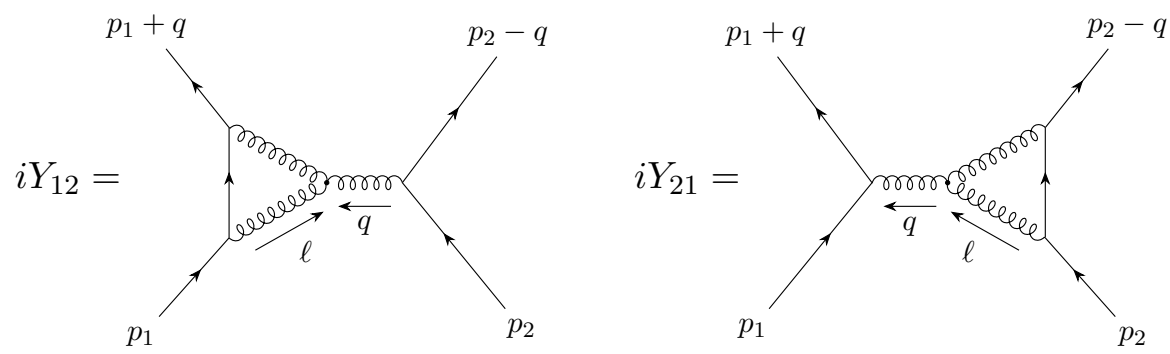

to contribute to the NLO observables. The series in $\hbar$ for these partial amplitudes contain terms at $\mathcal{O}\left(\hbar^{-2}\right)$; however, the decomposition of the full amplitude onto the colour basis in equation (3.25) shows that these always act as quantum corrections, and thus we need not calculate these diagrams.

\section{Colour deflection for $N$ particles}

Here we give the full general $N$ particle results for the colour deflection. The strategy to perform the calculation is iterative and follows ref. [11]. However, here we have not introduced an extra integration and performed a sum over integration labels as in [11]. As can be seen in equations (5.8), we are removing self-interactions. At NLO this leads to an important distinction between sums over particle species. Accordingly, in the following expressions we have separated the contributions according to the type of sum involved. 
Our result for the NLO colour deflection is

$$
\begin{aligned}
\Delta^{(2)} c_{\alpha}^{a}\left(\tau_{\alpha}\right)= & \sum_{\substack{\beta=1, \beta \neq \alpha \\
\gamma=1, \gamma \neq \alpha}}^{N} \int \hat{\mathrm{d}}^{4} \bar{q}_{2} \int \hat{\mathrm{d}}^{4} \bar{q}_{3} \hat{\delta}\left(\bar{q}_{3} \cdot u_{\gamma}\right) \hat{\delta}\left(\bar{q}_{2} \cdot u_{\beta}\right) e^{i \bar{q}_{3} \cdot b_{\gamma}} e^{i \bar{q}_{2} \cdot b_{\beta}} \\
& \times e^{-i\left(\bar{q}_{2}+\bar{q}_{3}\right) \cdot\left(b_{\alpha}+u_{\alpha} \tau_{\alpha}\right)} \mathcal{H}_{A}^{a,(2)}\left(\bar{q}_{1}, \bar{q}_{3} ; u_{\alpha}, u_{\gamma}, u_{\beta}\right) \\
& +\sum_{\substack{\beta=1, \beta \neq \alpha \\
\gamma=1, \gamma \neq \beta}}^{N} \int \hat{\mathrm{d}}^{4} \bar{q}_{2} \int \hat{\mathrm{d}}^{4} \bar{q}_{3} \hat{\delta}\left(\bar{q}_{3} \cdot u_{\gamma}\right) \hat{\delta}\left(\bar{q}_{2} \cdot u_{\beta}\right) e^{i \bar{q}_{3} \cdot b_{\gamma}} e^{i \bar{q}_{2} \cdot b_{\beta}} \\
& \times e^{-i\left(\bar{q}_{2}+\bar{q}_{3}\right) \cdot\left(b_{\alpha}+u_{\alpha} \tau_{\alpha}\right)} \mathcal{H}_{B}^{a,(2)}\left(\bar{q}_{1}, \bar{q}_{3} ; u_{\alpha}, u_{\gamma}, u_{\beta}\right)
\end{aligned}
$$

where $\bar{q}_{i j \ldots}=\bar{q}_{i}+\bar{q}_{j}+\cdots$ and

$$
\operatorname{tr}(A, B, C, D) \equiv \frac{1}{4} \operatorname{tr}(A \not B \not C \not D) .
$$

Here,

$$
\begin{aligned}
& \mathcal{H}_{A}^{a,(2)}\left(\bar{q}_{1}, \bar{q}_{3} ; u_{\alpha}, u_{\gamma}, u_{\beta}\right)= i \frac{f^{a b c} c_{\gamma}^{b} c_{\alpha}^{c} c_{\alpha} \cdot c_{\beta}}{m_{\alpha} \bar{q}_{2}^{2} \bar{q}_{3}^{2} \bar{q}_{23} \cdot u_{\alpha}\left(\bar{q}_{2} \cdot u_{\alpha}\right)^{2}} \\
& \times\left(u_{\alpha} \cdot u_{\gamma} \operatorname{tr}\left(\bar{q}_{2}, \bar{q}_{3}, u_{\alpha}, u_{\beta}\right)+\bar{q}_{2} \cdot u_{\alpha} \operatorname{tr}\left(\bar{q}_{2}, u_{\beta}, u_{\alpha}, u_{\gamma}\right)\right) \\
&-f^{a b c} f^{c d e} c_{\gamma}^{b} c_{\beta}^{d} c_{\alpha}^{e}\left(\frac{u_{\alpha} \cdot u_{\beta} u_{\alpha} \cdot u_{\gamma}}{\bar{q}_{2}^{2} \bar{q}_{3}^{2} \bar{q}_{2} \cdot u_{\alpha} \bar{q}_{23} \cdot u_{\alpha}}\right), \\
& \mathcal{H}_{B}^{a,(2)}\left(\bar{q}_{1}, \bar{q}_{3} ; u_{\alpha}, u_{\gamma}, u_{\beta}\right)= i \frac{f^{a b c} c_{\beta}^{b} c_{\alpha}^{c} c_{\beta} \cdot c_{\gamma}}{m_{\beta} \bar{q}_{3}^{2} \bar{q}_{23}^{2} \bar{q}_{23} \cdot u_{\alpha}\left(\bar{q}_{3} \cdot u_{\beta}\right)^{2}}\left(u_{\alpha} \cdot u_{\beta} \operatorname{tr}\left(\bar{q}_{23}, u_{\beta}, \bar{q}_{3}, u_{\gamma}\right)\right. \\
&\left.+\bar{q}_{3} \cdot u_{\beta} \operatorname{tr}\left(\bar{q}_{3}, u_{\alpha}, u_{\beta}, u_{\gamma}\right)\right)+\frac{f^{a b c} f^{b d e} c_{\alpha}^{c} c_{\gamma}^{d} c_{\beta}^{e}}{\bar{q}_{3}^{2} \bar{q}_{23}^{2} \bar{q}_{23} \cdot u_{\alpha}} \\
& \\
& \times\left(-\frac{u_{\beta} \cdot u_{\gamma} \bar{q}_{3} \cdot u_{\alpha}}{\bar{q}_{2}^{2}}+\frac{2 u_{\alpha} \cdot u_{\gamma} \bar{q}_{3} \cdot u_{\beta}}{\bar{q}_{2}^{2}}-\frac{u_{\alpha} \cdot u_{\beta} u_{\beta} \cdot u_{\gamma}}{\bar{q}_{3} \cdot u_{\beta}}\right) .
\end{aligned}
$$

In order to reach the form of the colour impulse in section 5 we set $N=2$ and perform the time integration on the support of the on-shell conditions. To recover the final observables we define the loop momentum as $\bar{\ell} \equiv \bar{q}_{3}$ and $\bar{\ell} \equiv \bar{q}_{2}+\bar{q}_{3}$ in the first and second contributions in (C.3) respectively.

Open Access. This article is distributed under the terms of the Creative Commons Attribution License (CC-BY 4.0), which permits any use, distribution and reproduction in any medium, provided the original author(s) and source are credited.

\section{References}

[1] S. Babak et al., Science with the space-based interferometer LISA. V: extreme mass-ratio inspirals, Phys. Rev. D 95 (2017) 103012 [arXiv: 1703.09722] [INSPIRE].

[2] J.F. Donoghue, Leading quantum correction to the Newtonian potential, Phys. Rev. Lett. 72 (1994) 2996 [gr-qc/9310024] [InSPIRE]. 
[3] J.F. Donoghue, General relativity as an effective field theory: the leading quantum corrections, Phys. Rev. D 50 (1994) 3874 [gr-qc/9405057] [INSPIRE].

[4] J.F. Donoghue and T. Torma, On the power counting of loop diagrams in general relativity, Phys. Rev. D 54 (1996) 4963 [hep-th/9602121] [INSPIRE].

[5] B.R. Holstein and J.F. Donoghue, Classical physics and quantum loops, Phys. Rev. Lett. 93 (2004) 201602 [hep-th/0405239] [INSPIRE].

[6] D. Neill and I.Z. Rothstein, Classical space-times from the $S$ matrix, Nucl. Phys. B $\mathbf{8 7 7}$ (2013) 177 [arXiv: 1304.7263] [INSPIRE].

[7] N.E.J. Bjerrum-Bohr, J.F. Donoghue and P. Vanhove, On-shell techniques and universal results in quantum gravity, JHEP 02 (2014) 111 [arXiv:1309.0804] [INSPIRE].

[8] R. Monteiro, D. O'Connell and C.D. White, Black holes and the double copy, JHEP 12 (2014) 056 [arXiv: 1410.0239] [INSPIRE].

[9] A. Luna, R. Monteiro, I. Nicholson, D. O'Connell and C.D. White, The double copy: bremsstrahlung and accelerating black holes, JHEP 06 (2016) 023 [arXiv:1603.05737] [INSPIRE].

[10] T. Damour, Gravitational scattering, post-Minkowskian approximation and effective one-body theory, Phys. Rev. D 94 (2016) 104015 [arXiv: 1609.00354] [INSPIRE].

[11] W.D. Goldberger and A.K. Ridgway, Radiation and the classical double copy for color charges, Phys. Rev. D 95 (2017) 125010 [arXiv:1611.03493] [InSPIRE].

[12] F. Cachazo and A. Guevara, Leading singularities and classical gravitational scattering, JHEP 02 (2020) 181 [arXiv: 1705.10262] [INSPIRE].

[13] A. Laddha and A. Sen, Gravity waves from soft theorem in general dimensions, JHEP 09 (2018) 105 [arXiv: 1801.07719] [inSPIRE].

[14] C. Cheung, I.Z. Rothstein and M.P. Solon, From scattering amplitudes to classical potentials in the post-Minkowskian expansion, Phys. Rev. Lett. 121 (2018) 251101 [arXiv: 1808.02489] [INSPIRE].

[15] D.A. Kosower, B. Maybee and D. O'Connell, Amplitudes, observables, and classical scattering, JHEP 02 (2019) 137 [arXiv:1811.10950] [INSPIRE].

[16] Z. Bern, C. Cheung, R. Roiban, C.-H. Shen, M.P. Solon and M. Zeng, Scattering amplitudes and the conservative Hamiltonian for binary systems at third post-Minkowskian order, Phys. Rev. Lett. 122 (2019) 201603 [arXiv:1901.04424] [INSPIRE].

[17] A. Antonelli, A. Buonanno, J. Steinhoff, M. van de Meent and J. Vines, Energetics of two-body Hamiltonians in post-Minkowskian gravity, Phys. Rev. D 99 (2019) 104004 [arXiv: 1901.07102] [INSPIRE].

[18] N.E.J. Bjerrum-Bohr, J.F. Donoghue, B.R. Holstein, L. Planté and P. Vanhove, Bending of light in quantum gravity, Phys. Rev. Lett. 114 (2015) 061301 [arXiv:1410.7590] [INSPIRE].

[19] N.E.J. Bjerrum-Bohr, B.R. Holstein, J.F. Donoghue, L. Planté and P. Vanhove, Illuminating light bending, PoS(CORFU2016) 077 (2017) [arXiv:1704.01624] [INSPIRE].

[20] T. Damour, High-energy gravitational scattering and the general relativistic two-body problem, Phys. Rev. D 97 (2018) 044038 [arXiv:1710.10599] [InSPIRE].

[21] A. Luna, I. Nicholson, D. O'Connell and C.D. White, Inelastic black hole scattering from charged scalar amplitudes, JHEP 03 (2018) 044 [arXiv:1711.03901] [INSPIRE]. 
[22] A. Laddha and A. Sen, Logarithmic terms in the soft expansion in four dimensions, JHEP 10 (2018) 056 [arXiv: 1804.09193] [INSPIRE].

[23] A. Laddha and A. Sen, Observational signature of the logarithmic terms in the soft graviton theorem, Phys. Rev. D 100 (2019) 024009 [arXiv: 1806.01872] [INSPIRE].

[24] N.E.J. Bjerrum-Bohr, P.H. Damgaard, G. Festuccia, L. Planté and P. Vanhove, General relativity from scattering amplitudes, Phys. Rev. Lett. 121 (2018) 171601 [arXiv: 1806.04920] [INSPIRE].

[25] B. Sahoo and A. Sen, Classical and quantum results on logarithmic terms in the soft theorem in four dimensions, JHEP 02 (2019) 086 [arXiv:1808.03288] [INSPIRE].

[26] A. Koemans Collado, P. Di Vecchia and R. Russo, Revisiting the second post-Minkowskian eikonal and the dynamics of binary black holes, Phys. Rev. D 100 (2019) 066028 [arXiv: 1904.02667] [INSPIRE].

[27] A. Brandhuber and G. Travaglini, On higher-derivative effects on the gravitational potential and particle bending, JHEP 01 (2020) 010 [arXiv:1905.05657] [INSPIRE].

[28] A. Cristofoli, N.E.J. Bjerrum-Bohr, P.H. Damgaard and P. Vanhove, Post-Minkowskian Hamiltonians in general relativity, Phys. Rev. D 100 (2019) 084040 [arXiv:1906.01579] [INSPIRE].

[29] A. Laddha and A. Sen, Classical proof of the classical soft graviton theorem in D $>4$, Phys. Rev. D 101 (2020) 084011 [arXiv: 1906.08288] [INSPIRE].

[30] Z. Bern, C. Cheung, R. Roiban, C.-H. Shen, M.P. Solon and M. Zeng, Black hole binary dynamics from the double copy and effective theory, JHEP 10 (2019) 206 [arXiv: 1908.01493] [INSPIRE].

[31] P.H. Damgaard, K. Haddad and A. Helset, Heavy black hole effective theory, JHEP 11 (2019) 070 [arXiv: 1908.10308] [InSPIRE].

[32] G. Kälin and R.A. Porto, From boundary data to bound states, JHEP 01 (2020) 072 [arXiv: 1910.03008] [INSPIRE].

[33] N.E.J. Bjerrum-Bohr, A. Cristofoli and P.H. Damgaard, Post-Minkowskian scattering angle in Einstein gravity, JHEP 08 (2020) 038 [arXiv:1910.09366] [INSPIRE].

[34] G. Kälin and R.A. Porto, From boundary data to bound states. Part II. Scattering angle to dynamical invariants (with twist), JHEP 02 (2020) 120 [arXiv:1911.09130] [INSPIRE].

[35] M. Accettulli Huber, A. Brandhuber, S. De Angelis and G. Travaglini, Note on the absence of $R^{2}$ corrections to Newton's potential, Phys. Rev. D 101 (2020) 046011 [arXiv: 1911.10108] [INSPIRE].

[36] A.P. Saha, B. Sahoo and A. Sen, Proof of the classical soft graviton theorem in $D=4$, JHEP 06 (2020) 153 [arXiv:1912.06413] [INSPIRE].

[37] R. Aoude, K. Haddad and A. Helset, On-shell heavy particle effective theories, JHEP 05 (2020) 051 [arXiv: 2001.09164] [InSPIRE].

[38] Z. Bern, H. Ita, J. Parra-Martinez and M.S. Ruf, Universality in the classical limit of massless gravitational scattering, Phys. Rev. Lett. 125 (2020) 031601 [arXiv:2002.02459] [INSPIRE].

[39] C. Cheung and M.P. Solon, Classical gravitational scattering at $\mathcal{O}\left(G^{3}\right)$ from Feynman diagrams, JHEP 06 (2020) 144 [arXiv:2003.08351] [INSPIRE]. 
[40] A. Cristofoli, P.H. Damgaard, P. Di Vecchia and C. Heissenberg, Second-order post-Minkowskian scattering in arbitrary dimensions, JHEP 07 (2020) 122 [arXiv: 2003.10274] [INSPIRE].

[41] Z. Bern, A. Luna, R. Roiban, C.-H. Shen and M. Zeng, Spinning black hole binary dynamics, scattering amplitudes and effective field theory, arXiv:2005.03071 [INSPIRE].

[42] J. Parra-Martinez, M.S. Ruf and M. Zeng, Extremal black hole scattering at $\mathcal{O}\left(G^{3}\right)$ : graviton dominance, eikonal exponentiation, and differential equations, JHEP 11 (2020) 023 [arXiv:2005. 04236] [INSPIRE].

[43] K. Haddad and A. Helset, The double copy for heavy particles, Phys. Rev. Lett. 125 (2020) 181603 [arXiv: 2005.13897] [INSPIRE].

[44] M. Accettulli Huber, A. Brandhuber, S. De Angelis and G. Travaglini, Eikonal phase matrix, deflection angle and time delay in effective field theories of gravity, Phys. Rev. D 102 (2020) 046014 [arXiv: 2006. 02375] [INSPIRE].

[45] C. Cheung and M.P. Solon, Tidal effects in the post-Minkowskian expansion, Phys. Rev. Lett. 125 (2020) 191601 [arXiv:2006.06665] [INSPIRE].

[46] M. A, D. Ghosh, A. Laddha and P.V. Athira, Soft radiation from scattering amplitudes revisited, arXiv: 2007.02077 [INSPIRE].

[47] G. Kälin, Z. Liu and R.A. Porto, Conservative dynamics of binary systems to third post-Minkowskian order from the effective field theory approach, arXiv:2007.04977 [INSPIRE].

[48] K. Haddad and A. Helset, Gravitational tidal effects in quantum field theory, arXiv: 2008.04920 [INSPIRE].

[49] G. Kälin, Z. Liu and R.A. Porto, Conservative tidal effects in compact binary systems to next-to-leading post-Minkowskian order, arXiv:2008.06047 [INSPIRE].

[50] B. Sahoo, Classical sub-subleading soft photon and soft graviton theorems in four spacetime dimensions, arXiv:2008.04376 [INSPIRE].

[51] P. Di Vecchia, C. Heissenberg, R. Russo and G. Veneziano, Universality of ultra-relativistic gravitational scattering, Phys. Lett. B 811 (2020) 135924 [arXiv:2008.12743] [INSPIRE].

[52] Z. Bern, J.J. Carrasco, M. Chiodaroli, H. Johansson and R. Roiban, The duality between color and kinematics and its applications, arXiv:1909.01358 [INSPIRE].

[53] A. Luna et al., Perturbative spacetimes from Yang-Mills theory, JHEP 04 (2017) 069 [arXiv: 1611.07508] [INSPIRE].

[54] W.D. Goldberger and A.K. Ridgway, Bound states and the classical double copy, Phys. Rev. D 97 (2018) 085019 [arXiv: 1711.09493] [inSPIRE].

[55] A. Luna, R. Monteiro, D. O'Connell and C.D. White, The classical double copy for Taub-NUT spacetime, Phys. Lett. B 750 (2015) 272 [arXiv:1507.01869] [INSPIRE].

[56] K. Lee, Kerr-Schild double field theory and classical double copy, JHEP 10 (2018) 027 [arXiv: 1807.08443] [INSPIRE].

[57] A. Luna, R. Monteiro, I. Nicholson and D. O'Connell, Type D spacetimes and the Weyl double copy, Class. Quant. Grav. 36 (2019) 065003 [arXiv: 1810.08183] [INSPIRE].

[58] T. Adamo, E. Casali, L. Mason and S. Nekovar, Plane wave backgrounds and colour-kinematics duality, JHEP 02 (2019) 198 [arXiv:1810.05115] [INSPIRE]. 
[59] M. Carrillo González, B. Melcher, K. Ratliff, S. Watson and C.D. White, The classical double copy in three spacetime dimensions, JHEP 07 (2019) 167 [arXiv:1904.11001] [INSPIRE].

[60] W. Cho and K. Lee, Heterotic Kerr-Schild double field theory and classical double copy, JHEP 07 (2019) 030 [arXiv:1904.11650] [InSPIRE].

[61] M. Carrillo González, R. Penco and M. Trodden, Shift symmetries, soft limits, and the double copy beyond leading order, Phys. Rev. D 102 (2020) 105011 [arXiv:1908.07531] [INSPIRE].

[62] N. Moynihan, Kerr-Newman from minimal coupling, JHEP 01 (2020) 014 [arXiv: 1909.05217] [INSPIRE].

[63] I. Bah, R. Dempsey and P. Weck, Kerr-Schild double copy and complex worldlines, JHEP 02 (2020) 180 [arXiv: 1910.04197] [InSPIRE].

[64] Y.-T. Huang, U. Kol and D. O'Connell, Double copy of electric-magnetic duality, Phys. Rev. D 102 (2020) 046005 [arXiv:1911.06318] [INSPIRE].

[65] R. Alawadhi, D.S. Berman, B. Spence and D. Peinador Veiga, S-duality and the double copy, JHEP 03 (2020) 059 [arXiv: 1911.06797] [INSPIRE].

[66] L. Borsten, I. Jubb, V. Makwana and S. Nagy, Gauge $\times$ gauge on spheres, JHEP 06 (2020) 096 [arXiv: 1911.12324] [INSPIRE].

[67] K. Kim, K. Lee, R. Monteiro, I. Nicholson and D. Peinador Veiga, The classical double copy of a point charge, JHEP 02 (2020) 046 [arXiv: 1912.02177] [INSPIRE].

[68] A. Banerjee, E.Ó. Colgáin, J.A. Rosabal and H. Yavartanoo, Ehlers as EM duality in the double copy, arXiv:1912.02597 [INSPIRE].

[69] N. Bahjat-Abbas, R. Stark-Muchão and C.D. White, Monopoles, shockwaves and the classical double copy, JHEP 04 (2020) 102 [arXiv:2001.09918] [INSPIRE].

[70] N. Moynihan and J. Murugan, On-shell electric-magnetic duality and the dual graviton, arXiv:2002.11085 [INSPIRE].

[71] T. Adamo, L. Mason and A. Sharma, MHV scattering of gluons and gravitons in chiral strong fields, Phys. Rev. Lett. 125 (2020) 041602 [arXiv:2003.13501] [INSPIRE].

[72] L. Alfonsi, C.D. White and S. Wikeley, Topology and Wilson lines: global aspects of the double copy, JHEP 07 (2020) 091 [arXiv:2004.07181] [INSPIRE].

[73] A. Luna, S. Nagy and C. White, The convolutional double copy: a case study with a point, JHEP 09 (2020) 062 [arXiv:2004.11254] [INSPIRE].

[74] C. Keeler, T. Manton and N. Monga, From Navier-Stokes to Maxwell via Einstein, JHEP 08 (2020) 147 [arXiv: 2005.04242] [INSPIRE].

[75] G. Elor, K. Farnsworth, M.L. Graesser and G. Herczeg, The Newman-Penrose map and the classical double copy, arXiv:2006.08630 [INSPIRE].

[76] A. Cristofoli, Gravitational shock waves and scattering amplitudes, arXiv:2006.08283 [INSPIRE].

[77] R. Alawadhi, D.S. Berman and B. Spence, Weyl doubling, JHEP 09 (2020) 127 [arXiv: 2007.03264] [INSPIRE]. 
[78] E. Casali and A. Puhm, A double copy for celestial amplitudes, arXiv:2007.15027 [INSPIRE].

[79] T. Adamo and A. Ilderton, Classical and quantum double copy of back-reaction, JHEP 09 (2020) 200 [arXiv: 2005.05807] [INSPIRE].

[80] D.A. Easson, C. Keeler and T. Manton, Classical double copy of nonsingular black holes, Phys. Rev. D 102 (2020) 086015 [arXiv:2007.16186] [InSPIRE].

[81] E. Chacón, H. García-Compeán, A. Luna, R. Monteiro and C.D. White, New heavenly double copies, arXiv:2008.09603 [INSPIRE].

[82] W.D. Goldberger, S.G. Prabhu and J.O. Thompson, Classical gluon and graviton radiation from the bi-adjoint scalar double copy, Phys. Rev. D 96 (2017) 065009 [arXiv:1705.09263] [INSPIRE].

[83] W.D. Goldberger, J. Li and S.G. Prabhu, Spinning particles, axion radiation, and the classical double copy, Phys. Rev. D 97 (2018) 105018 [arXiv:1712.09250] [InSPIRE].

[84] D. Chester, Radiative double copy for Einstein-Yang-Mills theory, Phys. Rev. D 97 (2018) 084025 [arXiv: 1712.08684] [INSPIRE].

[85] C.-H. Shen, Gravitational radiation from color-kinematics duality, JHEP 11 (2018) 162 [arXiv: 1806. 07388] [INSPIRE].

[86] J. Plefka, J. Steinhoff and W. Wormsbecher, Effective action of dilaton gravity as the classical double copy of Yang-Mills theory, Phys. Rev. D 99 (2019) 024021 [arXiv: 1807.09859] [inSPIRE].

[87] J. Plefka, C. Shi, J. Steinhoff and T. Wang, Breakdown of the classical double copy for the effective action of dilaton-gravity at NNLO, Phys. Rev. D 100 (2019) 086006 [arXiv:1906.05875] [INSPIRE].

[88] A.P.V. and A. Manu, Classical double copy from color kinematics duality: a proof in the soft limit, Phys. Rev. D 101 (2020) 046014 [arXiv:1907.10021] [INSPIRE].

[89] G.L. Almeida, S. Foffa and R. Sturani, Classical gravitational self-energy from double copy, arXiv: 2008.06195 [INSPIRE].

[90] V. Vaidya, Gravitational spin Hamiltonians from the S matrix, Phys. Rev. D 91 (2015) 024017 [arXiv: 1410.5348] [INSPIRE].

[91] A. Guevara, Holomorphic classical limit for spin effects in gravitational and electromagnetic scattering, JHEP 04 (2019) 033 [arXiv:1706.02314] [INSPIRE].

[92] A. Guevara, A. Ochirov and J. Vines, Scattering of spinning black holes from exponentiated soft factors, JHEP 09 (2019) 056 [arXiv:1812.06895] [INSPIRE].

[93] M.-Z. Chung, Y.-T. Huang, J.-W. Kim and S. Lee, The simplest massive S-matrix: from minimal coupling to black holes, JHEP 04 (2019) 156 [arXiv:1812.08752] [INSPIRE].

[94] Y.F. Bautista and A. Guevara, From scattering amplitudes to classical physics: universality, double copy and soft theorems, arXiv:1903.12419 [INSPIRE].

[95] B. Maybee, D. O'Connell and J. Vines, Observables and amplitudes for spinning particles and black holes, JHEP 12 (2019) 156 [arXiv:1906.09260] [INSPIRE].

[96] A. Guevara, A. Ochirov and J. Vines, Black-hole scattering with general spin directions from minimal-coupling amplitudes, Phys. Rev. D 100 (2019) 104024 [arXiv:1906.10071] [INSPIRE]. 
[97] N. Arkani-Hamed, Y.-T. Huang and D. O'Connell, Kerr black holes as elementary particles, JHEP 01 (2020) 046 [arXiv: 1906.10100] [INSPIRE].

[98] Y.F. Bautista and A. Guevara, On the double copy for spinning matter, arXiv:1908.11349 [INSPIRE].

[99] L. de la Cruz, A. Kniss and S. Weinzierl, Double copies of fermions as matter that interacts only gravitationally, Phys. Rev. Lett. 116 (2016) 201601 [arXiv:1601.04523] [INSPIRE].

[100] M.-Z. Chung, Y.-T. Huang and J.-W. Kim, Classical potential for general spinning bodies, JHEP 09 (2020) 074 [arXiv: 1908.08463] [INSPIRE].

[101] M.-Z. Chung, Y.-T. Huang and J.-W. Kim, Kerr-Newman stress-tensor from minimal coupling to all orders in spin, arXiv:1911.12775 [INSPIRE].

[102] M.-Z. Chung, Y.-T. Huang, J.-W. Kim and S. Lee, Complete Hamiltonian for spinning binary systems at first post-Minkowskian order, JHEP 05 (2020) 105 [arXiv:2003.06600] [INSPIRE].

[103] M. Levi, A.J. Mcleod and M. Von Hippel, NNNLO gravitational quadratic-in-spin interactions at the quartic order in $G$, arXiv:2003.07890 [INSPIRE].

[104] R. Aoude, M.-Z. Chung, Y.-T. Huang, C.S. Machado and M.-K. Tam, The silence of binary Kerr, Phys. Rev. Lett. 125 (2020) 181602 [arXiv:2007.09486] [INSPIRE].

[105] S.K. Wong, Field and particle equations for the classical Yang-Mills field and particles with isotopic spin, Nuovo Cim. A 65 (1970) 689 [INSPIRE].

[106] A. Strominger, Asymptotic symmetries of Yang-Mills theory, JHEP 07 (2014) 151 [arXiv: 1308.0589] [INSPIRE].

[107] M. Pate, A.-M. Raclariu and A. Strominger, Color memory: a Yang-Mills analog of gravitational wave memory, Phys. Rev. Lett. 119 (2017) 261602 [arXiv:1707.08016] [INSPIRE].

[108] A. Strominger, Lectures on the infrared structure of gravity and gauge theory, arXiv: 1703.05448 [INSPIRE].

[109] A. Campoleoni, D. Francia and C. Heissenberg, Asymptotic charges at null infinity in any dimension, Universe 4 (2018) 47 [arXiv:1712.09591] [INSPIRE].

[110] T. He and P. Mitra, Asymptotic symmetries in $(d+2)$-dimensional gauge theories, JHEP 10 (2019) 277 [arXiv: 1903.03607] [InSPIRE].

[111] R. Gonzo, T. Mc Loughlin, D. Medrano and A. Spiering, Asymptotic charges and coherent states in $Q C D$, arXiv:1906.11763 [INSPIRE].

[112] A. Campoleoni, D. Francia and C. Heissenberg, Electromagnetic and color memory in even dimensions, Phys. Rev. D 100 (2019) 085015 [arXiv:1907.05187] [INSPIRE].

[113] U.W. Heinz, Kinetic theory for non-Abelian plasmas, Phys. Rev. Lett. 51 (1983) 351 [INSPIRE].

[114] U.W. Heinz, A relativistic colored spinning particle in an external color field, Phys. Lett. B 144 (1984) 228 [INSPIRE].

[115] U.W. Heinz, Quark-gluon transport theory. Part 1. The classical theory, Annals Phys. 161 (1985) 48 [INSPIRE]. 
[116] U.W. Heinz, Quark-gluon transport theory. Part 2. Color response and color correlations in a quark-gluon plasma, Annals Phys. 168 (1986) 148 [INSPIRE].

[117] H.-T. Elze and U.W. Heinz, Quark-gluon transport theory, Phys. Rept. 183 (1989) 81 [INSPIRE].

[118] D.F. Litim and C. Manuel, Semiclassical transport theory for non-Abelian plasmas, Phys. Rept. 364 (2002) 451 [hep-ph/0110104] [INSPIRE].

[119] L.D. McLerran and R. Venugopalan, Computing quark and gluon distribution functions for very large nuclei, Phys. Rev. D 49 (1994) 2233 [hep-ph/9309289] [InSPIRE].

[120] L.D. McLerran and R. Venugopalan, Gluon distribution functions for very large nuclei at small transverse momentum, Phys. Rev. D 49 (1994) 3352 [hep-ph/9311205] [INSPIRE].

[121] E. Iancu and R. Venugopalan, The color glass condensate and high-energy scattering in $Q C D$, in Quark-gluon plasma 4, R.C. Hwa and X.-N. Wang eds., World Scientific, Singapore (2003), pg. 249 [hep-ph/0303204] [INSPIRE].

[122] K. Kajantie, L.D. McLerran and R. Paatelainen, Gluon radiation from a classical point particle, Phys. Rev. D 100 (2019) 054011 [arXiv: 1903.01381] [INSPIRE].

[123] K. Kajantie, L.D. McLerran and R. Paatelainen, Gluon radiation from a classical point particle II: dense gluon fields, Phys. Rev. D 101 (2020) 054012 [arXiv:1911.12738] [INSPIRE].

[124] L.G. Yaffe, Large N limits as classical mechanics, Rev. Mod. Phys. 54 (1982) 407 [inSPIRE].

[125] G. Kaiser, Phase space approach to relativistic quantum mechanics. 1. Coherent state representation for massive scalar particles, J. Math. Phys. 18 (1977) 952 [INSPIRE].

[126] S. Twareque Ali and J. Antoine, Coherent states of the $1+1$ dimensional Poincaré group: square integrability and a relativistic Weyl transform, Ann. Inst. H. Poincaré Phys. Theor. 51 (1989) 23.

[127] K. Kowalski, J. Rembieliński and J.-P. Gazeau, On the coherent states for a relativistic scalar particle, Annals Phys. 399 (2018) 204 [arXiv: 1903.07312] [INSPIRE].

[128] A.M. Perelomov, Coherent states for arbitrary Lie group, Commun. Math. Phys. 26 (1972) 222 [math-ph/0203002].

[129] A. Perelomov, Generalized coherent states and their applications, Springer, Berlin, Heidelberg, Germany (1986) [INSPIRE].

[130] M. Combescure and D. Robert, Coherent states and applications in mathematical physics, Springer, Dordrecht, The Netherlands (2012).

[131] J.J. Sakurai and J. Napolitano, Modern quantum mechanics, Cambridge University Press, Cambridge, U.K. (2017).

[132] M. Mathur and D. Sen, Coherent states for SU(3), J. Math. Phys. 42 (2001) 4181 [quant-ph/0012099] [INSPIRE].

[133] M. Mathur and H.S. Mani, SU(N) coherent states, J. Math. Phys. 43 (2002) 5351 [quant-ph/0206005] [INSPIRE].

[134] M. Mathur, I. Raychowdhury and R. Anishetty, SU(N) irreducible Schwinger bosons, J. Math. Phys. 51 (2010) 093504 [arXiv:1003.5487] [INSPIRE]. 
[135] M. Mathur and I. Raychowdhury, $\mathrm{SU}(N)$ coherent states and irreducible Schwinger bosons, J. Phys. A 44 (2011) 035203 [arXiv: 1007.1510] [INSPIRE].

[136] A. Ochirov and B. Page, Multi-quark colour decompositions from unitarity, JHEP 10 (2019) 058 [arXiv: 1908.02695] [INSPIRE].

[137] M. Lüscher, Asymptotic behavior of classical Yang-Mills fields in Minkowski space, Nucl. Phys. B 140 (1978) 429 [inSPIRE].

[138] T. Adamo and E. Casali, Perturbative gauge theory at null infinity, Phys. Rev. D 91 (2015) 125022 [arXiv: 1504.02304] [INSPIRE].

[139] G. Alexanian, A.P. Balachandran, G. Immirzi and B. Ydri, Fuzzy CP2, J. Geom. Phys. 42 (2002) 28 [hep-th/0103023] [INSPIRE].

[140] H. Grosse and H. Steinacker, Finite gauge theory on fuzzy $C P^{2}$, Nucl. Phys. B 707 (2005) 145 [hep-th/0407089] [INSPIRE].

[141] J. Ellis, TikZ-Feynman: Feynman diagrams with TikZ, Comput. Phys. Commun. 210 (2017) 103 [arXiv: 1601.05437] [INSPIRE]. 